No. 586

April 2018

A monolithic conservative level set method with built-in redistancing

M. Quezada de Luna, D. Kuzmin, C. E. Kees

ISSN: 2190-1767 


\title{
A monolithic conservative level set method with built-in redistancing
}

\author{
Manuel Quezada de Luna * Dmitri Kuzmin ${ }^{\dagger} \quad$ Christopher E. Kees ${ }^{\ddagger}$
}

\begin{abstract}
We introduce a new level set method for representing evolving interfaces. In the case of divergence-free velocity fields, the new method satisfies a conservation principle. Conservation is important for many applications such as modeling two-phase incompressible flow. In the present implementation, the conserved quantity is defined as the integral of a smoothed characteristic function. The new approach embeds level sets into a volume of fluid formulation. The evolution of an approximate signed distance function is governed by a conservation law for its (smoothed) sign. The non-linear level set transport equation is regularized by adding a flux correction term that assures a non-singular Jacobian and penalizes deviations from a distance function. The result is a locally conservative level set method with built-in elliptic redistancing. The continuous model is monolithic in the sense that the level set transport model, the volume of fluid law of mass conservation, and the minimization problem that preserves the approximate distance function property are incorporated into a single equation. There is no need for any extra stabilization, artificial compression, flux limiting, redistancing, mass correction, and other numerical fixes which are commonly used in level set or volume of fluid methods. In addition, there is just one free parameter that controls the strength of regularization and penalization in the model. The accuracy and conservation properties of the monolithic finite element / level set method are illustrated by the results of numerical studies for passive advection of free interfaces.
\end{abstract}

\section{Introduction}

Flows of immiscible fluids with different material properties (e.g. water and air) commonly occur in fluid mechanics and engineering applications. The design of numerical simulation techniques for such multi-phase flows requires an accurate mathematical description of evolving interfaces between subdomains occupied by different fluids. Moreover, numerical methods should be efficient and preserve important qualitative properties of the continuous model as far as possible. This work is motived by the requirement of mass and volume conservation in numerical models of incompressible two-phase flows.

There is an extensive list of methods based on reconstruction of material interfaces from auxiliary functions. Popular choices include the Volume of Fluid (VOF) method by Hirt and Nichols [17] and level set techniques by Osher and Sethian [30], Sussman et al. [36]. The VOF method uses a characteristic function to identify the phases, e.g., fluids A and B. The exact phase indicator function equals one in fluid A, zero in fluid B, and is discontinuous at the interface. The average over any given cell defines the fraction of the cell occupied by fluid A. Therefore, cells with averages in the range $(0,1)$ must contain the interface. The characteristic function is evolved by solving a hyperbolic conservation law. In applications to interfacial two-phase flows, the velocity field is obtained by solving the incompressible Navier-Stokes equations with piecewise-constant density and viscosity. Therefore, a reconstruction of the interface from cell averages is required to determine the instantaneous location of the boundary between the two phases and set the material properties inside each subdomain.

\footnotetext{
*US Army, ERDC-CHL. manuel.q.deluna@usace.army.mil

${ }^{\dagger}$ Institute of Applied Mathematics (LS III), TU Dortmund University. kuzmin@math.uni-dortmund.de

${ }^{\ddagger}$ US Army, ERDC-CHL. christopher.e.kees@usace.army.mil
} 
The level set method reconstructs the interface between the two phases using a continuous auxiliary function which has a constant value on the evolving interface (e.g., a signed distance function which is positive in fluid A and negative in fluid B). Since the interface is defined as the manifold on which the prescribed value (e.g., zero) is attained, the process of interface reconstruction reduces to calculation of isolines/isosurfaces. The level set function is advected by the fluid velocity and remains constant along the characteristics of the hyperbolic transport equation which models its evolution.

The main disadvantage of the VOF method is the difficulty and ambiguity of interface reconstruction. In level set methods, interfaces are uniquely defined and easy to reconstruct. However, numerical approximations based on the level set approach may fail to preserve the area/volume of an incompressible fluid enclosed by the interface. This issue is commonly dealt with by using global mass correction techniques like the one introduced by Smolianski [34]. Additionally, a variety of hybrid methods based on combined VOF and level set formulations were proposed in the literature, see for instance Enright et al. [14], Ianniello and Di Mascio [19], Sussman and Puckett [35]. Kees et al. [20] used the VOF solution to correct the redistanced level set function so as to enforce a regularized conservation law for a smoothed Heaviside function. This approach guarantees local mass conservation since the amount of correction depends on the residual of the conservation law. The underlying design philosophy is similar to that behind the dual level set method of Lesage and Dervieux [24] but the use of a Laplacian-type regularization term leads to a well-posed non-linear problem with just one free parameter. Building on this conservative level set algorithm, optimal control approaches to flux-based mass correction were developed in Basting and Kuzmin [7], Kuzmin [22]. As shown in these papers, the use of PDE-constrained optimization makes it possible to minimize deviations from a non-conservative target subject to a flux-corrected conservation law for the composition of the Heaviside function and the corrected level set function. However, the practical utility of such mass correction techniques is limited by the high cost of solving non-linear constrained optimization problems.

In this work, we present a new monolithic level set algorithm for mass-conserving evolution of approximate distance functions. The proposed methodology is based on a single VOF-like non-linear conservation law for the composition of a smoothed sign function and a level set function. Similarly to Kees et al. [20], an elliptic regularization term is introduced to control the mass flux and prevent the Jacobian of the non-linear system from becoming singular away from the interface. Moreover, we show that this term can be designed to penalize deviations from a distance function in the same manner as in Basting and Kuzmin [6], Li et al. [26]. As an additional benefit, the embedding of such penalization into the conservation law eliminates the need for any stabilization of advective terms.

The rest of this paper is organized as follows: In $\S 2$ we introduce some notation and preliminaries. In particular, we define the finite element spaces, the basis functions for the spatial discretization, and regularized versions of characteristic-like functions that we use. In $\S 2.3$ we review the non-conservative level set method and commonly employed redistancing techniques. The conservative level set method of Kees et al. [20] is presented in $\S 2.4$ and used as a starting point for the derivation of the first monolithic version in $\S 3$. The level set formulation derived in this section already contains the key ingredients but is quite sensitive to the choice of redistancing operators and iterative solvers for the non-linear problem. We cure this lack of robustness in $\S 4$ by using a (lumped-mass) $L^{2}$ projection of the target for the penalization term. In $\S 5$ we illustrate the convergence behavior of the proposed monolithic conservative level set method combined with $\mathbb{P}_{1}$ and $\mathbb{P}_{2}$ finite element approximations. In $\S 6$ we perform additional numerical studies for two- and three-dimensional benchmark problems. Finally, in $\S 7$ we summarize the outcomes of this work, make concluding remarks, and outline some potential directions for further improvements of the proposed methodology. 


\section{Preliminaries}

\subsection{Spatial discretization}

We use the continuous Galerkin finite element method to discretize all equations in space. Let $d=$ $\{1,2,3\}$ be the number of space dimensions and $\Omega \subset \mathbb{R}^{d}$ be a bounded domain with boundary $\partial \Omega \subset$ $\mathbb{R}^{d-1}$ which we decompose into $\partial \Omega^{-}=\{\mathbf{x} \in \partial \Omega \mid \mathbf{u} \cdot \mathbf{n}<0\}$ and $\partial \Omega^{+}=\{\mathbf{x} \in \partial \Omega \mid \mathbf{u} \cdot \mathbf{n} \geq 0\}$. Timedependent variables are defined on the time interval $t \in[0, T]$, where $T>0$. Given a computational mesh $\mathcal{T}_{h}$, we consider the finite element space $X_{h}^{p}=\left\{w \in \mathcal{C}^{0}(\Omega)|w|_{K} \in \mathbb{P}_{\mathrm{p}}, \forall K \in \mathcal{T}_{h}\right\}$ spanned by basis functions $\left\{w_{1}, \ldots, w_{\operatorname{dim}\left(X_{h}^{p}\right)}\right\}$ which possess the partition of unity property; i.e., $\sum_{j} w_{j}(\mathbf{x})=1$. The degrees of freedom associated with these basis functions are denoted by uppercase letters. The finite element solution $u_{h}(\mathbf{x}) \in X_{h}$ is given by $u_{h}(\mathbf{x})=\sum_{j \in \mathcal{I}\left(\Omega_{i}\right)} U_{j} w_{j}(\mathbf{x})$, where $\Omega_{i}$ is the patch of elements containing node $i$. Here, and in the rest of this paper, the notation $\mathcal{I}(z)$ is used for the index set containing the numbers of all basis functions whose support on $z$ is of nonzero measure.

\subsection{Use of regularized characteristic-like functions}

In different parts of this work, we employ characteristic-like functions. In particular, local conservation laws are formulated in terms of the Heaviside and sign functions. We replace such discontinuous functions by regularized counterparts, as is common in level set methods (e.g. Fedkiw and Osher [16], Kees et al. [20]). We define the regularized Heaviside function $H_{\epsilon}(\phi)$ and the regularized sign function $S_{\epsilon}(\phi)$ as follows:

$$
\begin{aligned}
& H_{\epsilon}(\phi)= \begin{cases}0, & \text { if } \phi \leq-\epsilon, \\
\frac{1}{2}\left(1+\frac{\phi}{\epsilon}+\frac{1}{\pi} \sin \left(\pi \frac{\phi}{\epsilon}\right)\right), & \text { if }-\epsilon<\phi<\epsilon, \\
1, & \text { if } \phi \geq \epsilon,\end{cases} \\
& S_{\epsilon}(\phi)=2 H_{\epsilon}(\phi)-1 .
\end{aligned}
$$

The value of the parameter $\epsilon>0$ determines the width of the smooth transition zone between two constant states. Fedkiw and Osher [16] use $\epsilon=\frac{3}{2} h(\mathbf{x})$, where $h(\mathbf{x})$ is a suitably defined local mesh size. We follow this definition in all numerical experiments of this paper unless stated otherwise. As the mesh is refined, the regularized characteristic-like functions (1a) and (1b) converge to the sharp Heaviside function $H(\phi)=\lim _{\epsilon \rightarrow 0} H_{\epsilon}(\phi)$ and the sharp sign function $S(\phi)=\lim _{\epsilon \rightarrow 0} S_{\epsilon}(\phi)$, respectively.

In the context of two-phase flows, $H_{\epsilon}(\phi)$ is the local volume fraction of the fluid whose characteristic function is $H(\phi)$. We remark that diffuse interface approximations of this kind are employed, e.g., in phase field methods and in the conservative level set method of Olsson and Kreiss [29].

\subsection{Non-conservative level set method and redistancing}

Level set approaches belong to the class of front-capturing methods which are based on implicit representations of evolving interfaces. A continuous function $\phi(\cdot, t) \in \mathcal{C}^{0}(\bar{\Omega})$ is used to represent the interface as it moves through the flow field. The evolution of $\phi(\mathbf{x}, t)$ is governed by the first-order hyperbolic equation

$$
\partial_{t} \phi+\mathbf{v} \cdot \nabla \phi=0
$$

where $\mathbf{v}(\mathbf{x}, t)$ is an extension of the interface velocity to the entire domain, see, for example, Fedkiw and Osher [16], Sethian [33]. The choice of extension velocity is not unique away from the material interface, but in this work we assume it is in fact the material velocity and is divergence free. In the classical level set formulation of Osher and Sethian [30], the initial condition is given by a signed distance $\phi(\mathbf{x}, 0)= \pm \operatorname{dist}(\mathbf{x}, \Gamma(0))$, and the position of an interface at time $t \geq 0$ corresponds to the 
zero level set $\Gamma(t)=\{\mathbf{x} \in \Omega \mid \phi(\mathbf{x}, t)=0\}$. It is common knowledge that the solution of (2) remains constant along each characteristic $\mathbf{x}(t)$ defined by the ordinary differential equation $\frac{d \mathbf{x}(t)}{d t}=\mathbf{v}(\mathbf{x}(t), t)$ and an initial condition of the form $\mathbf{x}(0)=\mathbf{x}_{0}$. That is, equation (2) is the Eulerian form of the Lagrangian identity $\phi(\mathbf{x}(t), t))=\phi\left(\mathbf{x}_{0}, 0\right) \forall t \geq 0$. It follows that $\mathbf{x}(t) \in \Gamma(t)$ whenever $\mathbf{x}_{0} \in \Gamma(0)$.

At the continuous level, the regularized Heaviside function $H_{\epsilon}(\phi)$ remains constant along the characteristics as well. Using the fact that $\nabla \cdot \mathbf{v}=0$, we find that the solution of (2) must satisfy

$$
\partial_{t}\left(H_{\epsilon}(\phi)\right)+\nabla \cdot\left(\mathbf{v} H_{\epsilon}(\phi)\right)=0
$$

Integrating over $\Omega$ and using the divergence theorem, we obtain the integral conservation law

$$
\frac{d}{d t} \int_{\Omega} H_{\epsilon}(\phi) d \mathbf{x}+\int_{\partial \Omega} H_{\epsilon}(\phi) \mathbf{v} \cdot \mathbf{n} d \mathbf{s}=0
$$

By virtue of $(1 b)$, conservation of $H_{\epsilon}$ implies conservation of $S_{\epsilon}$ and vice versa. Hence, physicscompatible numerical approximations should conserve $H_{\epsilon}$ and $S_{\epsilon}$ at the discrete level.

We use the continuous Galerkin finite element method to discretize (2) in space. Due to the lack of coercivity, the linear form associated with the advective term $\mathbf{v} \cdot \nabla \phi$ needs to be stabilized (see, e.g., Ern and Guermond [15]). If the velocity field is exactly divergence-free, the numerical solution $\phi_{h}$ produced by the (stabilized) Galerkin method is globally conservative in the sense that

$$
\frac{d}{d t} \int_{\Omega} \phi_{h} d \mathbf{x}+\int_{\partial \Omega} \phi_{h} \mathbf{v} \cdot \mathbf{n} d \mathbf{s}=0 .
$$

However, this conservation property has no physical significance and does not imply that

$$
\frac{d}{d t} \int_{\Omega} H_{\epsilon}\left(\phi_{h}\right) d \mathbf{x}+\int_{\partial \Omega} H_{\epsilon}\left(\phi_{h}\right) \mathbf{v} \cdot \mathbf{n} d \mathbf{s}=0
$$

In this work, we call any level set algorithm that may violate (5) non-conservative. Furthermore, we do not distinguish between mass conservation, volume conservation, and phase conservation in the context of level set methods for incompressible two-phase flows.

Another desirable property that tends to be lost in the process of level set advection is the signed distance function (SDF) property of the initial condition. Even the exact solution of (2) does not preserve it for general velocity fields. As a consequence, the gradients of $\phi_{h}$ may become very steep or very flat, leading to numerical instabilities or ill-conditioned interface reconstruction problems. A variety of redistancing techniques have been developed to restore the SDF property while preserving the zero level set as far as possible. Efficient geometric approaches to level set reinitialization were proposed, e.g., by Ausas et al. [3], Sethian [32, 33], Tsai et al. [39]. PDE-based redistancing procedures use numerical methods to solve the non-linear Eikonal equation $|\nabla \phi|= \pm 1$ or the Euler-Lagrange equation of a minimization problem. A review of such PDE-based approaches can be found, e.g., in Basting and Kuzmin [6]. Depending on the type of subproblems to be solved at each iteration or pseudo-time step they can be classified into hyperbolic, parabolic, and elliptic ones.

The hyperbolic redistancing method of Sussman et al. [36] initializes $\phi$ by a level set function $\tilde{\phi}$ that defines the desired position of the interface but does not possess the SDF property. In the process of redistancing, $\phi$ is advanced in pseudo-time by solving the non-linear transport equation

$$
\partial_{\tau} \phi+\operatorname{sgn}(\tilde{\phi}) \frac{\nabla \phi}{|\nabla \phi|} \cdot \nabla \phi=\operatorname{sgn}(\tilde{\phi}) .
$$

Note that the steady state solution of this hyperbolic PDE satisfies the Eikonal equation and has the same zero level set as $\tilde{\phi}$. In a practical implementation, just a few pseudo-time steps are needed to reinitialize $\phi$ in a narrow band around the interface where large deviations from a SDF would have particularly harmful consequences. It is also possible to embed this kind of redistancing into the 
transport equation (2), as proposed in Ville et al. [41], see also Bonito et al. [9]. One-step algorithms that update and re-distance $\phi$ in this way are referred to as convected level set methods.

Level set functions with desired properties can also be generated by minimizing a suitably defined objective functional. The redistancing procedures proposed by Li et al. [26] and Basting and Kuzmin [6] replace the Eikonal equation by the Euler-Lagrange equation

$$
\frac{\partial \mathcal{R}(\phi)}{\partial \phi}=0
$$

of a minimization problem for a generic objective functional $\mathcal{R}(\phi)$. For example, definition

$$
\mathcal{R}(\phi)=\frac{1}{2} \int_{\Omega}(|\nabla \phi|-1)^{2} d \mathbf{x}
$$

leads to a least squares problem for the residual of the Eikonal equation. Li et al. [26] solve this non-linear problem using time marching based on the parabolic transport equation

$$
\partial_{\tau} \phi-\nabla \cdot\left(1-\frac{1}{|\nabla \phi|}\right) \nabla \phi=0
$$

Basting and Kuzmin [6] solve the elliptic Euler-Lagrange equation using a fixed-point iteration method instead of time marching. To assure well-posedness and prevent any significant displacements of the interface $\Gamma(\tilde{\phi})=\{\mathbf{x} \in \Omega \mid \tilde{\phi}(\mathbf{x}, t)=0\}$, they augment $\mathcal{R}(\phi)$ by the penalty term

$$
\mathcal{P}(\phi, \tilde{\phi})=\frac{\alpha}{2} \int_{\Gamma(\tilde{\phi})} \phi^{2} d \mathbf{s}
$$

where $\alpha \gg 1$ is a parameter that determines the amount of penalization. The variational elliptic problem associated with the penalized version of the Eikonal functional (6) is given by

$$
\int_{\Omega}\left(1-\frac{1}{\left|\nabla \phi_{h}\right|}\right) \nabla \phi_{h} \cdot \nabla w d \mathbf{x}+\alpha \int_{\Gamma\left(\tilde{\phi_{h}}\right)} \phi_{h} w d \mathbf{s}=0, \quad \forall w \in X_{h},
$$

where $X_{h}$ is the space of admissible test functions. It is also possible to minimize other functionals $\mathcal{R}(\phi)$ and incorporate problem-specific features into the definition of the potential function. In particular, the redistancing procedure may be configured to produce a constant function rather than a SDF near local extrema. The rationale for using such objective functionals is explained in §3.1.

The minimization-based approach to redistancing is the first key ingredient of the methodology to be developed in the present work. In $\S 4$, we embed generic Euler-Lagrange equations into a conservation law for the regularized Heaviside function $H_{\epsilon}(\phi)$ in a manner similar to that in which the signed Eikonal equation is embedded into the transport equation of a convected level set method. Building on ideas developed in Basting and Kuzmin [6], Kees et al. [20], Li et al. [26], Ville et al. [41], we design a level set algorithm that preserves desired properties of level set functions as they evolve in contrast to predictor-corrector approaches based on advection, redistancing, and mass correction.

\subsection{Conservative level set method}

The second key ingredient of the proposed property-preserving monolithic scheme is a built-in mass correction procedure based on the work of Kees et al. [20]. Their conservative level set method is a predictor-corrector algorithm which involves four stages. For simplicity, we define these stages using the forward Euler approximation of time derivatives but the use of other time integrators is possible and appropriate for many applications of practical interest. 
Let $\phi^{n}$ denote a numerical approximation to the level set function at the time level $t^{n}=n \Delta t$. The initial condition $\phi^{0}$ can be defined as an approximate signed distance function of the interface $\Gamma(0)$. The four stages that are used to update $\phi^{n}$ in the algorithm of Kees et al. [20] are as follows:

Stage 1: Non-conservative level set (NCLS) advection. Given a divergence-free velocity field $\mathbf{v}$, advance $\phi^{n}$ in time by solving the level set transport equation

$$
\frac{\tilde{\phi}^{n+1}-\phi^{n}}{\Delta t}+\mathbf{v} \cdot \nabla \phi^{n}=0 .
$$

Stage 2: Redistancing. Post-process the advected level set function $\tilde{\phi}^{n+1}$ using one of the methods presented in $\S 2.3$ to produce a redistanced non-conservative level set function $\hat{\phi}^{n+1}$.

Stage 3: Volume of fluid (VOF) advection. Solve the scalar conservation law

$$
\frac{\hat{H}^{n+1}-H_{\epsilon}\left(\phi^{n}\right)}{\Delta t}+\nabla \cdot\left(\mathbf{v} H_{\epsilon}\left(\phi^{n}\right)\right)=0
$$

to calculate a mass-conserving approximate Heaviside function $\hat{H}^{n+1}$ as in VOF-like methods.

Stage 4: Mass correction. Adjust $\hat{\phi}^{n+1}$ by adding a localized correction $u^{n+1}$ such that

$$
\begin{aligned}
H_{\epsilon}\left(\hat{\phi}^{n+1}+u^{n+1}\right)-\hat{H}^{n+1}-\kappa h \Delta u^{n+1} & =0, \\
\left.\nabla u^{n+1} \cdot \mathbf{n}\right|_{\partial \Omega} & =0, \\
\phi^{n+1} & =\hat{\phi}^{n+1}+u^{n+1},
\end{aligned}
$$

where $\kappa>0$ is a free parameter and $h$ is the mesh size. The regularization term $\kappa h \Delta u^{n+1}$ in (9a) assures that a conservation law holds for $H_{\epsilon}\left(\phi^{n+1}\right)$ and $\phi^{n+1}$ is uniquely defined away from the interface.

The mass conservation property of the fractional-step level set method follows from the fact that

$$
\int_{\Omega} \hat{H}_{h}^{n+1} d \mathbf{x}=\int_{\Omega} H_{\epsilon}\left(\phi_{h}^{n}\right) d \mathbf{x}
$$

(as long as $\mathbf{v} \cdot \mathbf{n}=0$ or $H_{\epsilon}\left(\phi_{h}^{n}\right)=0$ on the outer boundary $\partial \Omega$ ) and

$$
\int_{\Omega} H_{\epsilon}\left({\hat{\phi_{h}}}^{n+1}+u_{h}^{n+1}\right) d \mathbf{x}=\int_{\Omega} \hat{H}_{h}^{n+1} d \mathbf{x}
$$

due to the boundary condition (9b). By definition of $\hat{H}^{n+1}$, equation (9a) can be written as

$$
\Delta u^{n+1}=\frac{\Delta t}{\kappa h}\left(\frac{H_{\epsilon}\left(\phi^{n+1}\right)-H_{\epsilon}\left(\phi^{n}\right)}{\Delta t}+\nabla \cdot\left(\mathbf{v} H_{\epsilon}\left(\phi^{n}\right)\right)\right) .
$$

Hence, the amount of mass correction is proportional to the residual of a local conservation law.

The type of space discretization can be chosen individually for each stage. Kees et al. [20] discretize all transport equations using continuous finite elements and streamline upwind Petrov-Galerkin (SUPG) stabilization, as proposed by Brooks and Hughes [11].

\subsubsection{Towards a monolithic conservative level set method}

The main objective of this work is to convert the fractional-step method presented in $\S 2.4$ into a singlestage level set algorithm. As a first step towards that end, we combine stages 3 and 4 . Substituting $u^{n+1}=\phi^{n+1}-\hat{\phi}^{n+1}$ into the conservation law (10) and boundary condition (9b), we obtain

$$
\begin{aligned}
\frac{H_{\epsilon}\left(\phi^{n+1}\right)-H_{\epsilon}\left(\phi^{n}\right)}{\Delta t}+\nabla \cdot\left(\mathbf{v} H_{\epsilon}\left(\phi^{n}\right)-\frac{\kappa h}{\Delta t} \nabla\left(\phi^{n+1}-\hat{\phi}^{n+1}\right)\right) & =0, \\
\left.\nabla\left(\phi^{n+1}-\hat{\phi}^{n+1}\right) \cdot \mathbf{n}\right|_{\partial \Omega} & =0 .
\end{aligned}
$$


This formulation makes it possible to determine $\phi^{n+1}$ without calculating the auxiliary functions $\hat{H}^{n+1}$ and $u^{n+1}$. We also remark that there is no need for any extra stabilization of the advective term when it comes to solving equation (11) numerically. In contrast to a finite element approximation $\hat{H}_{h}^{n+1}$ produced by a stabilized Galerkin discretization of (8), the regularized Heaviside function $H_{\epsilon}\left(\phi_{h}^{n+1}\right)$ is always bounded by 0 and 1 . Therefore, no violations of the discrete maximum principle can occur even if equation (11) is discretized in space using the standard continuous Galerkin method. While Touré and Soulaïmani [38] have proposed a monolithic level set scheme that combine interface transport and redistancing, we know of no other work with the features outlined above.

\section{Monolithic conservative level set method}

Representation (11) of the conservative level set method reveals that the regularization term penalizes deviations of $\nabla \phi^{n+1}$ from $\nabla \hat{\phi}^{n+1}$ in regions where $H_{\epsilon}$ is constant. If $\hat{\phi}^{n+1}$ is an approximate distance function, then the mass correction procedure based on (11) will preserve the SDF property as far as possible. Instead of using an auxiliary solution $\hat{\phi}^{n+1}$ as a target, the regularization term may be designed to penalize a suitably chosen redistancing potential in the process of level set evolution. As a first level set algorithm based on a monolithic formulation of this kind, we consider

$$
\partial_{t} S_{\epsilon}(\phi)+\nabla \cdot\left[\mathbf{v} S_{\epsilon}(\phi)-\lambda\left(1-\frac{1}{|\nabla \phi|}\right) \nabla \phi\right]=0
$$

where $\lambda$ is a free parameter. We impose the following initial and boundary conditions:

$$
\begin{aligned}
S_{\epsilon}(\phi(\mathbf{x}, 0)) & =S_{\epsilon}\left(\phi_{0}(\mathbf{x})\right) & & \forall \mathbf{x} \in \Omega, \\
\left(1-\frac{1}{|\nabla \phi|}\right) \nabla \phi \cdot \mathbf{n} & =0 & & \forall \mathbf{x} \in \partial \Omega .
\end{aligned}
$$

Formulation (12) combines the conservative level set method of Kees et al. [20] with the elliptic redistancing procedure of Basting and Kuzmin [6]. As we discuss below, this particular form of the regularization term still has some drawbacks. However, the above non-linear problem already exhibits the essential features of a monolithic conservative level set algorithm with built-in redistancing.

Remark 3.0.1 (On the use of the sign function). Note that we use the regularized sign function $S_{\epsilon}(\phi)$, as defined in §2.2, instead of the regularized Heaviside function $H_{\epsilon}(\phi)$. This form of the conservation law is more symmetric and treats both phases equally. Since - $\phi$ satisfies (12) as well, the position of the interface remains unchanged if the sign of the initial condition is reversed.

Remark 3.0.2 (Conservation). We discretize (12) in space using a continuous Galerkin finite element approximation. In view of (12c), the variational form of the non-linear problem is given by

$$
\int_{\Omega} \partial_{t} S_{\epsilon}\left(\phi_{h}\right) w d x-\int_{\Omega}\left[\mathbf{v} S_{\epsilon}\left(\phi_{h}\right)-\lambda\left(1-\frac{1}{\left|\nabla \phi_{h}\right|}\right) \nabla \phi_{h}\right] \cdot \nabla w d x+\int_{\partial \Omega} S_{\epsilon}\left(\phi_{h}\right) w \mathbf{v} \cdot \mathbf{n} d \mathbf{s}=0, \quad \forall w \in X_{h} .
$$

Substituting the test function $w \equiv 1$, we obtain

$$
\int_{\Omega} \partial_{t} S_{\epsilon}\left(\phi_{h}\right) d x+\int_{\partial \Omega} S_{\epsilon}\left(\phi_{h}\right) \mathbf{v} \cdot \mathbf{n} d \mathbf{s}=0 \Longrightarrow \int_{\Omega} \partial_{t} H_{\epsilon}\left(\phi_{h}\right) d x+\int_{\partial \Omega} H_{\epsilon}\left(\phi_{h}\right) \mathbf{v} \cdot \mathbf{n} d \mathbf{s}=0 .
$$

In other words, the Galerkin variational form (12) is globally conservative in the sense discussed in §2.3. Any consistent finite element discretization of (13) is conservative as well since $w \equiv 1$ is an admissible test function. Hence, mass conservation errors become arbitrarily small as the residuals of the non-linear discrete problem are driven to machine zero using an iterative solution procedure. 


\subsection{Single- vs. double well-potential functionals}

As discussed in $\S 2.3$, the parabolic redistancing method of Li et al. [26] and the elliptic redistancing method of Basting and Kuzmin [6] are derived by minimizing an appropriate functional

$$
\mathcal{R}(\phi)=\int_{\Omega} p(|\nabla \phi|) d \mathbf{x}
$$

The first-order optimality condition

$$
\left.\frac{\partial R(\phi+\varepsilon w)}{\partial \varepsilon}\right|_{\varepsilon \rightarrow 0}=0, \quad \forall w \in X
$$

implies that the solution $\phi$ of the minimization problem must satisfy

$$
\int_{\Omega} d_{p}\left(\left|\nabla \phi_{h}\right|\right) \nabla \phi_{h} \cdot \nabla w d \mathbf{x}=0, \quad \forall w \in X_{h}
$$

where

$$
d_{p}(|\nabla \phi|)=\frac{p^{\prime}(|\nabla \phi|)}{|\nabla \phi|}
$$

is the diffusion rate corresponding to the given potential function $p(|\nabla \phi|)$. In the context of elliptic redistancing, spurious displacements of the original zero level set are prevented by adding a penalty term (see §2.3). The possibility of using different potential functions makes it possible to control the outcome of the redistancing process depending on the desired geometric properties of $\phi$. In particular, it is important to ensure correct behavior in situations when $|\nabla \phi| \approx 0$; i.e., in the vicinity of local extrema or in regions where the level set function is (almost) constant.

The extension of the monolithic method (12) to a general potential function $p(|\nabla \phi|)$ is given by

$$
\begin{aligned}
\partial_{t} S_{\epsilon}(\phi)+\nabla \cdot\left[\mathbf{v} S_{\epsilon}(\phi)-\lambda d_{p}(|\nabla \phi|) \nabla \phi\right] & =0, \\
\left.d_{p}(|\nabla \phi|) \nabla \phi \cdot \mathbf{n}\right|_{\partial \Omega} & =0 .
\end{aligned}
$$

Given an appropriate finite element space $X_{h}$, the continuous Galerkin space discretization of equation (15) yields a numerical approximation $\phi_{h} \in X_{h}$ such that

$$
\begin{array}{r}
R\left(\phi_{h}, w\right):=\int_{\Omega} \partial_{t} S_{\epsilon}\left(\phi_{h}\right) w d \mathbf{x}-\int_{\Omega} \mathbf{v} S_{\epsilon}\left(\phi_{h}\right) \cdot \nabla w d \mathbf{x}+\int_{\partial \Omega} S_{\epsilon}\left(\phi_{h}\right) w \mathbf{v} \cdot \mathbf{n} d \mathbf{s} \\
+\int_{\Omega} \lambda d_{p}\left(\left|\nabla \phi_{h}\right|\right) \nabla \phi_{h} \cdot \nabla w d \mathbf{x}=0, \quad \forall w \in X_{h} .
\end{array}
$$

Li et al. [26] considered the potential functions

$$
\begin{aligned}
& p_{1}(s)=\frac{1}{2}(s-1)^{2} \quad \Longrightarrow \quad d_{1}(s)=1-\frac{1}{s}, \\
& p_{2}(s)=\left\{\begin{array}{ll}
\frac{1-\cos (2 \pi s)}{(2 \pi)^{2}}, & \text { if } s \leq 1, \\
\frac{1}{2}(s-1)^{2}, & \text { if } s>1
\end{array} \quad \Longrightarrow \quad d_{2}(s)= \begin{cases}\frac{\sin (2 \pi s)}{2 \pi s}, & \text { if } s \leq 1, \\
1-\frac{1}{s}, & \text { if } s>1 .\end{cases} \right.
\end{aligned}
$$

Basting and Kuzmin [6] found that the definition

$$
p_{3}(s)=\left\{\begin{array}{ll}
\frac{1}{2} s^{2}(s-1)^{2}, & \text { if } s \leq 1, \\
\frac{1}{2}(s-1)^{2}, & \text { if } s>1
\end{array} \quad \Longrightarrow \quad d_{3}(s)= \begin{cases}2 s^{2}-3 s+1, & \text { if } s \leq 1, \\
1-\frac{1}{s}, & \text { if } s>1\end{cases}\right.
$$

is better suited for their elliptic redistancing scheme. In Figure 1 we show the plots of the three different potential functions and the corresponding diffusion rates. 

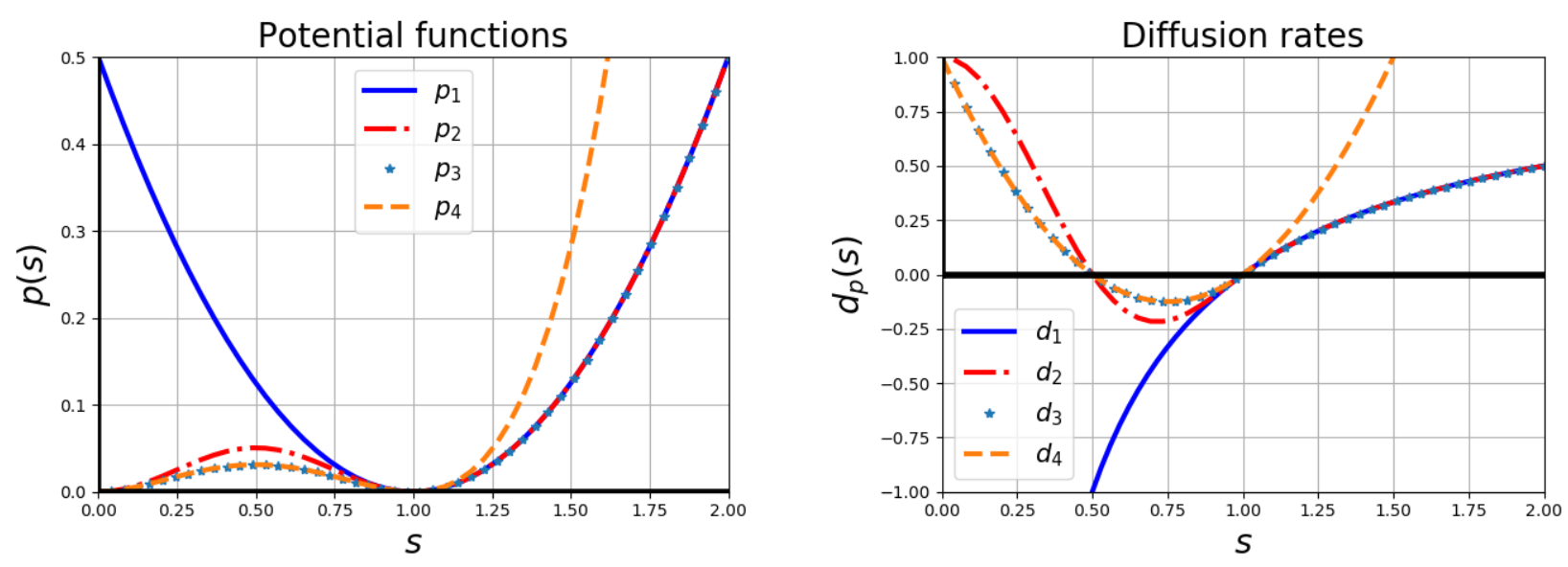

Figure 1: Potential functions defined by (17) and the corresponding diffusion rates.

We first remark that all of the above potential functions have a stable potential at $|\nabla \phi|=1$, while $p_{2}$ and $p_{3}$ have another one at $|\nabla \phi|=0$. It is also important to consider the behavior of the diffusion rate when $|\nabla \phi|<1$. In the case of $p_{1}$, we have $d_{1} \rightarrow-\infty$ when $|\nabla \phi| \rightarrow 0$; i.e., an infinite amount of anti-diffusion is applied in an attempt to correct the gradient if the level set profile becomes flat. Therefore, this potential is unlikely to behave properly in the presence of local extrema or in applications to truncated distance functions. This issue is extensively discussed by Utz et al. [40].

The diffusion rates corresponding to $p_{2}$ and $p_{3}$ are positive and finite when $|\nabla \phi|=0$. Therefore, moderate diffusion is introduced at local extrema and in flat regions. Instead of $p_{3}$, we employ

$$
p_{4}(s)=\frac{1}{2} s^{2}(s-1)^{2}, \quad \Longrightarrow \quad d_{4}(s)=2 s^{2}-3 s+1,
$$

which corresponds to the first branch of $p_{3}$. The plot for $p_{4}$ and the corresponding diffusion rate are also shown in Figure 1. Note that $p_{4}$ exhibits the correct behavior; i.e., it has the same stable potentials as $p_{2}$ and $p_{3}$, and its diffusion rate behaves as desired. Indeed, the only important difference compared to $p_{3}$ is that $p_{4}$ is more aggressive when $|\nabla \phi|>1$; i.e., stronger diffusion is applied when the gradient becomes too steep. The advantage of using $p_{4}$ lies in the fact that its diffusion rate is a polynomial with respect to $|\nabla \phi|$ and not a composite function. Therefore, it is easier to obtain a suitable approximation of the Jacobian corresponding to (16). In this work, we only consider $p_{1}$ and $p_{4}$, which are hereafter referred to as single- and double-well potential functions, respectively.

Remark 3.1.1 (About the different terms). To facilitate further discussion of the proposed methodology, we name the terms that appear in equation (15) as follows. The first two terms $\partial_{t} S_{\epsilon}(\phi)$ and $\nabla \cdot\left(\mathbf{v} S_{\epsilon}(\phi)\right)$ correspond to the time derivative and the advection term, respectively. The sum of these terms models the advection of the volume fraction by the velocity field $\mathbf{v}$. Regardless of whether the single- or double-well potential is employed in (15), the flux function of the built-in redistancing operator includes a linear part of the form $-\nabla \cdot(\lambda \nabla \phi)$. This diffusive component ensures non-singularity of the Jacobian corresponding to (16). Hence, we call it the regularization term. The remaining components of the redistancing operator in (15) penalize deviations from the distance function. Therefore, we refer to them as penalization terms. Note that the exact form of these terms depends on the choice of the potential function. For the single-well potential, the penalization term is given by $\nabla \cdot\left(\lambda \frac{\nabla \phi}{|\nabla \phi|}\right)$, while the penalization terms associated with the double-well potential are $\nabla \cdot\left[\lambda\left(2|\nabla \phi|^{2}-3|\nabla \phi|\right) \nabla \phi\right]$.

\subsection{Time integration and iterative solution}

Now that we have defined the potential functions, we can discretize the non-linear problem (16) in time and use it to evolve the finite element approximation $\phi_{h} \in X_{h}$. In all numerical experiments of 
this section, we use first order accurate time discretizations. The fully implicit one is given by

$$
\begin{aligned}
R\left(\phi_{h}, w\right) & :=\int_{\Omega} \frac{\left[S_{\epsilon}\left(\phi_{h}^{n+1}\right)-S_{\epsilon}\left(\phi_{h}^{n}\right)\right] w d \mathbf{x}}{\Delta t}-\int_{\Omega} \mathbf{v}^{n+1} S_{\epsilon}\left(\phi_{h}^{n+1}\right) \cdot \nabla w d \mathbf{x}+\int_{\partial \Omega} S_{\epsilon}\left(\phi_{h}^{n+1}\right) w \mathbf{v}^{n+1} \cdot \mathbf{n} d \mathbf{s} \\
& +\int_{\Omega} \lambda\left[\nabla \phi_{h}^{n+1}-\mathbf{q}\left(\phi_{h}^{n+1}\right)\right] \cdot \nabla w d \mathbf{x}=0, \quad \forall w \in X_{h},
\end{aligned}
$$

where $\mathbf{q}$ is the penalization term that depends on the choice of the potential function. In Section 4, we replace it by a $\mathcal{C}^{0}$ reconstruction $\mathbf{q}_{h}$. First and second order accurate time discretizations of the resulting mixed finite element approximation are presented in $\S 4.2$ and $\S 4.3$, respectively.

To solve the non-linear system produced by an implicit time discretization of (16), we use a (quasi-) Newton method. Consequently, we need to provide (an approximation of) the Jacobian containing the partial derivatives of $R\left(\phi_{h}, w_{i}\right)$. To regularize the term $|\nabla \phi|$ we approximate it by

$$
|\nabla \phi|_{\delta}:=\sqrt{\nabla \phi \cdot \nabla \phi+\delta^{2}}
$$

as proposed by Badia and Bonilla [4], Barrenechea et al. [5]. We use $\delta^{2}=10^{-15}$ in all numerical experiments. Assuming that all terms are treated implicitly as in (18), we consider the following approximation $J_{i j}^{k} \approx \frac{\partial R\left(\phi_{h}^{k}, w_{i}\right)}{\partial \Phi_{j}}$ of the Jacobian at the $k$-th Newton iteration:

$$
J_{i j}^{k}=\int_{\Omega} S_{\epsilon}^{\prime}\left(\phi_{h}^{k}\right)\left[\frac{1}{\Delta t} w_{i} w_{j}-\nabla w_{i} \cdot\left(\mathbf{v}^{n+1} w_{j}\right)\right] d \mathbf{x}+\int_{\partial \Omega} S_{\epsilon}^{\prime}\left(\phi_{h}^{k}\right) w_{i} w_{j} \mathbf{v}^{n+1} \cdot \mathbf{n} d \mathbf{x}+\mathcal{L}_{i j}^{k},
$$

where

$$
\mathcal{L}_{i j}^{k}=\int_{\Omega} \lambda \max \left(10^{-10}, 1-\frac{1}{\left|\nabla \phi_{h}^{k}\right|_{\delta}}\right) \nabla w_{i} \cdot \nabla w_{j} d \mathbf{x}+\int_{\Omega} \lambda \frac{\left(\nabla \phi_{h}^{k} \cdot \nabla w_{i}\right)\left(\nabla \phi_{h}^{k} \cdot \nabla w_{j}\right) d \mathbf{x}}{\left|\nabla \phi_{h}\right|_{\delta}^{3}},
$$

if the single-well potential is employed and

$$
\begin{aligned}
\mathcal{L}_{i j}^{k} & =\int_{\Omega} \lambda \max \left(10^{-10}, 1+2\left|\nabla \phi_{h}^{k}\right|^{2}-3\left|\nabla \phi_{h}^{k}\right|\right) \nabla w_{i} \cdot \nabla w_{j} d \mathbf{x} \\
& +\int_{\Omega} \lambda \max \left(10^{-10}, 4-\frac{3}{\left|\nabla \phi_{h}^{k}\right|_{\delta}}\right)\left(\nabla \phi_{h}^{k} \cdot \nabla w_{i}\right)\left(\nabla \phi_{h}^{k} \cdot \nabla w_{j}\right) d \mathbf{x}
\end{aligned}
$$

otherwise. For time discretizations with some terms treated explicitly, we use reduced versions of (19).

The regularization term must be treated implicitly in the single- and double-well potential version alike. The advection and the penalization terms can be treated explicitly or implicitly. To explore the numerical behavior of these alternatives, we consider the periodic vortex problem (see Rider and Kothe [31]) in the domain $\Omega=(0,1)^{2}$. The initial condition and velocity field are given by

$$
\begin{aligned}
\phi(\mathbf{x}, 0) & = \pm \operatorname{dist}\left(\mathbf{x}, \Gamma_{0}\right) \\
\mathbf{v}(x, y, t) & =\left[\begin{array}{c}
-\sin (\pi x)^{2} \sin (2 \pi y) \sin (2 \pi t / 8) \\
\sin (2 \pi x) \sin (\pi y)^{2} \sin (2 \pi t / 8)
\end{array}\right]
\end{aligned}
$$

where $\Gamma_{0}:=\left\{(x, y) \in \Omega \mid\left(x-x_{c}\right)^{2}+\left(y-y_{c}\right)^{2}=r^{2}\right\}$ is a circle of radius $r=0.15$ centered at $\left(x_{c}, y_{c}\right)=(0.5,0.75)$. We select the positive distance in $(20 \mathrm{a})$ if $\mathbf{x}=(x, y)$ is inside the circle $\Gamma_{0}$ and the negative distance otherwise. In Figure 2, we show the numerical solution at $t=2$ obtained using the single- and double-well potentials. These results indicate that explicit treatment of the advection term may give rise to significant distortions of evolving level sets, whereas the penalization term may be treated explicitly without producing such side effects. It is worth mentioning that implicit treatment of the penalization term is more challenging in the context of Newton-like methods. Indeed, the inclusion of this term makes the Jacobian more complicated and requires the use of certain approximations to 
avoid the possibility of having negative entries (see (19)). Alternatively, the contribution of the penalization term to the Jacobian may be omitted, as in the fixed-point iteration method proposed by Basting and Kuzmin [6]. In our experience, this approximation leads to a large number of Newton iterations per time step when the residual needs to be zero to machine precision. In the context of elliptic redistancing, the cost of solving the non-linear system remains acceptable because there is no need to use very small tolerances for the residuals. As shown by Basting and Kuzmin [6], a few fixed-point iterations are sufficient to restore the approximate signed distance function property.

Exp/Exp with single-well pot.

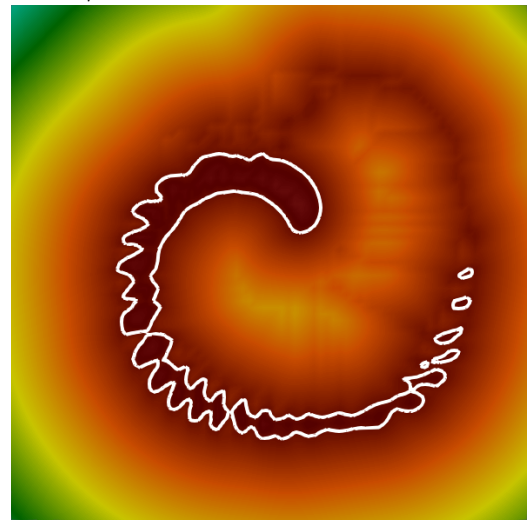

Imp/Exp with single-well pot.

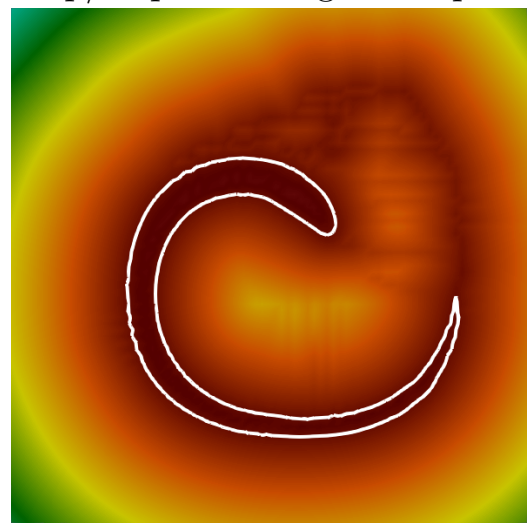

Exp/Exp with double-well pot.

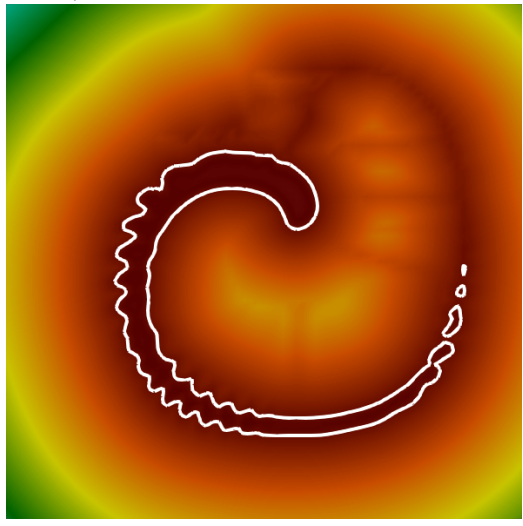

Imp/Exp with double-well pot.

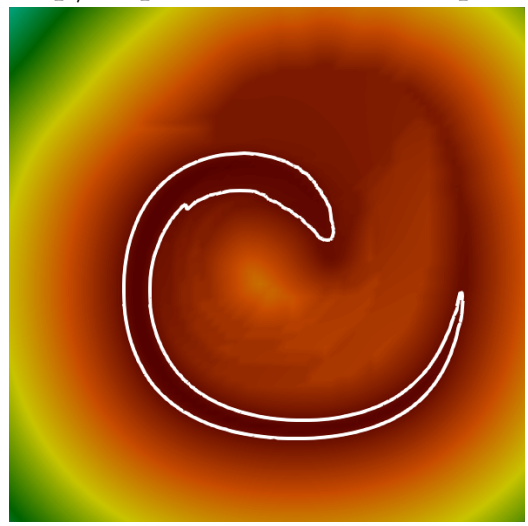

Exp/Imp with double-well pot.

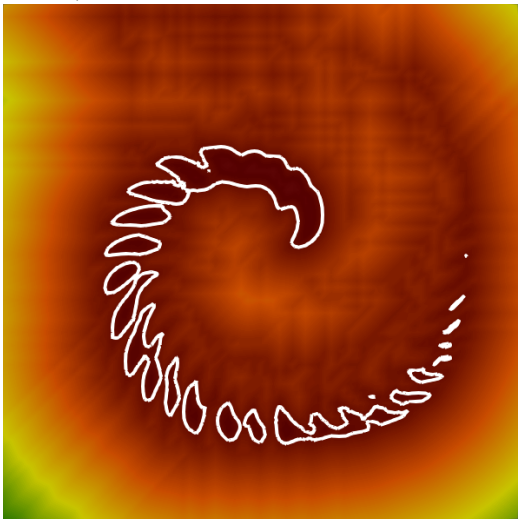

Imp/Imp with double-well pot.

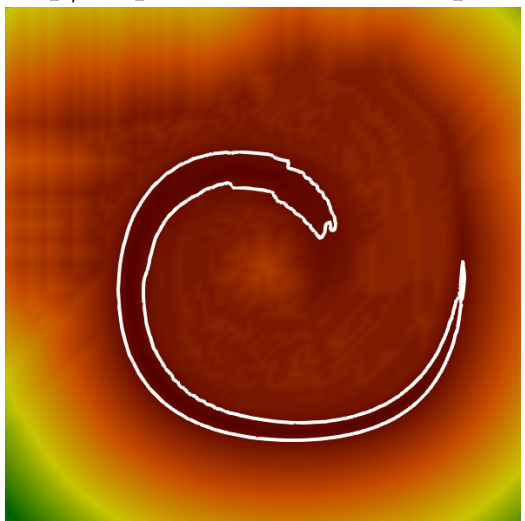

Figure 2: Solutions of the vortex problem at $t=2$ obtained using the monolithic level set formulation (15) with $\lambda=0.1$ and different potential functions. The advection term is treated explicitly in the first row and implicitly in the second row. The left panels correspond to the single-well potential with the penalization term treated explicitly. The panels in the middle and right correspond to the double-well potential with the penalization term treated explicitly and implicitly, respectively.

\section{Monolithic conservative level set method with $\mathcal{C}^{0}$ reconstruction}

Instead of using double-well potential functions, numerical difficulties associated with the presence of local extrema can be avoided by using a mixed finite element approximation of the terms that penalize deviations from a distance function. In this section, we consider the single-well potential version of the monolithic conservative level set method (15) and approximate the penalizing flux $\mathbf{q}$ using a regularized lumped-mass $L^{2}$ projection $\mathbf{q}_{h}$. The proposed approach is based on the methodology developed by 
Chan et al. [12] for iterative solution of the total variation regularization problem

$$
\alpha K^{*}\left(K u-u_{0}\right)-\nabla \cdot \frac{\nabla \phi}{|\nabla \phi|}=0
$$

where $K$ is a compact operator, $\alpha \in \mathbb{R}$ is a free parameter, and $u_{0}$ is a given target function. The regularized mixed form of this non-linear elliptic problem is given by

$$
\begin{aligned}
\alpha K^{*}\left(K u-u_{0}\right)-\nabla \cdot \mathbf{q} & =0, \\
\sqrt{|\nabla \phi|^{2}+\delta^{2}} \mathbf{q} & =\nabla \phi .
\end{aligned}
$$

Note that the auxiliary flux variable $\mathbf{q}$ is a continuous and well-behaved vector field. Moreover, the finite element discretization of (22) leads to a non-linear problem which can be solved efficiently.

Adapting the above idea to our problem, we write the conservation law (15) of the single-well potential monolithic level set method in the regularized mixed form

$$
\begin{aligned}
\partial_{t} S_{\epsilon}(\phi)+\nabla \cdot\left[\mathbf{v} S_{\epsilon}(\phi)-\lambda(\nabla \phi-\mathbf{q})\right] & =0, \\
\sqrt{|\nabla \phi|^{2}+\delta^{2}} \mathbf{q} & =\nabla \phi
\end{aligned}
$$

and impose the following initial and boundary conditions:

$$
\begin{aligned}
S_{\epsilon}(\phi(\mathbf{x}, 0)) & =S_{\epsilon}\left(\phi_{0}(\mathbf{x})\right), & & \forall \mathbf{x} \in \Omega, \\
(\nabla \phi-\mathbf{q}) \cdot \mathbf{n} & =0, & & \forall \mathbf{x} \in \partial \Omega .
\end{aligned}
$$

Integrating by parts in the weak form of (24a) and substituting the natural boundary condition (24d) into the resulting surface integral, we obtain the variational formulation

$$
\int_{\Omega} \partial_{t} S_{\epsilon}\left(\phi_{h}\right) w d \mathbf{x}-\int_{\Omega}\left[\mathbf{v} S_{\epsilon}\left(\phi_{h}\right)-\lambda\left(\nabla \phi_{h}-\mathbf{q}_{h}\right)\right] \cdot \nabla w d \mathbf{x}+\int_{\partial \Omega} S_{\epsilon}\left(\phi_{h}\right) w \mathbf{v} \cdot \mathbf{n} d \mathbf{x}=0 \quad \forall w \in X_{h}
$$

The variational form of equation (24b) corresponds to the weighted $L^{2}$ projection

$$
\int_{\Omega} \sqrt{\left|\nabla \phi_{h}\right|^{2}+\delta^{2}} \mathbf{q}_{h} w d \mathbf{x}=\int_{\Omega} \nabla \phi_{h} w d \mathbf{x} \quad \forall w \in X_{h} .
$$

Remark 4.0.1 (Conservation). Substituting $w \equiv 1$ into equation (25), we find that

$$
\int_{\Omega} \partial_{t} S_{\epsilon}\left(\phi_{h}\right) d \mathbf{x}+\int_{\partial \Omega} S_{\epsilon}\left(\phi_{h}\right) \mathbf{v} \cdot \mathbf{n} d \mathbf{s}=0
$$

which implies global conservation in the sense discussed in §2.3. See also remark 3.0.2.

Remark 4.0.2 (Incompressibility). In this paper, we assume that $\nabla \cdot \mathbf{v}=0$ and the interface $\Gamma(t)$ does not intersect the outer boundary $\partial \Omega$ at any time. That is, the regularized sign function $S_{\epsilon}\left(\phi_{h}\right)$ is constant on $\partial \Omega$. Hence, $\int_{\partial \Omega} S_{\epsilon}\left(\phi_{h}\right) \mathbf{v} \cdot \mathbf{n} d \mathbf{s}= \pm \int_{\partial \Omega} \mathbf{v} \cdot \mathbf{n} d \mathbf{s}=0$ for incompressible two-phase flows.

Remark 4.0.3 (On the choice of the parameter $\lambda$ ). The parameter $\lambda$ has units of velocity and can be non-dimensionalized using scaling by $h(\mathbf{x}) / \Delta t$, where $h(\mathbf{x})$ is a suitable measure of the local mesh size. Note that $h(\mathbf{x}) / \Delta t \sim 1$ if a CFL condition holds. In the below numerical study, we use

$$
\lambda=\tilde{\lambda} \frac{h(\mathbf{x})}{\left\|\phi_{h}-\bar{\phi}_{h}\right\|_{L^{\infty}(\Omega)}},
$$

where $\tilde{\lambda} \sim 1$ has units of velocity and $\bar{\phi}_{h}=\frac{1}{|\Omega|} \int_{\Omega} \phi_{h} d \mathbf{x}$. This definition of $\lambda$ ensures that the regularization and penalization terms vanish as $h(\mathbf{x}) \rightarrow 0$. We use $\tilde{\lambda}=1.0$ in all experiments of $\S 6$. 


\subsection{Finite element discretization}

We discretize (25) and (26) in space using the continuous Galerkin method. Given a finite element solution $\phi_{h} \in X_{h}$ of (25), the discrete version of (26) defines an equal-order $\mathcal{C}^{0}$ reconstruction

$$
\mathbf{q}_{h}\left(\phi_{h}\right)=\sum_{j} \mathbf{Q}_{j}\left(\phi_{h}\right) w_{j}(\mathbf{x})
$$

of the globally defined normal. In our present implementation of the monolithic level set method, the components of $\mathbf{Q}_{j} \in \mathbb{R}^{d}, j \in \mathcal{I}(\Omega)$ are calculated using the lumped diagonal form of the weighted mass matrix in the discrete version of (26). This approximation leads to the explicit formula

$$
Q_{i}^{(k)}\left(\phi_{h}\right)=\frac{\int_{\Omega} \partial_{k} \phi_{h} w_{i} d \mathbf{x}}{\int_{\Omega} \sqrt{\left|\nabla \phi_{h}\right|^{2}+\delta^{2}} w_{i} d \mathbf{x}}
$$

for the coefficients of $q_{h}^{(k)}=\sum_{j} Q_{j}^{(k)} w_{j}, k=\{1, \ldots, d\}$. We use the parameter setting $\delta^{2}=10^{-15}$ in all simulations. Whereas Chan et al. [12] used the mixed form (22) of (21) to speed up the non-linear solver, their far-reaching idea offers additional advantages in our context. In contrast to $\frac{\nabla \phi_{h}}{\left|\nabla \phi_{h}\right|}$, the projected penalization flux $\mathbf{q}_{h}$ is continuous and approaches zero smoothly as $\left|\nabla \phi_{h}\right| \rightarrow 0$. This property implies that penalization is automatically deactivated in the vicinity of local extrema, where the distance function property is impossible to be enforced by adding anti-diffusive corrections. Consequently, there is no need to use the double-well potential with this level set formulation.

\subsection{First-order time discretization}

Given a continuous Galerkin discretization of equation (25), we consider a first-order implicit time stepping scheme which updates the finite element solution $\phi_{h} \in X_{h}$ as follows:

$$
\begin{aligned}
R\left(\phi_{h}, w\right):=\int_{\Omega} \frac{\left[S_{\epsilon}\left(\phi_{h}^{n+1}\right)-S_{\epsilon}\left(\phi_{h}^{n}\right)\right] w d \mathbf{x}}{\Delta t}-\int_{\Omega} & \left(S_{\epsilon}\left(\phi_{h}^{n+1}\right) \mathbf{v}^{n+1}-\lambda\left(\nabla \phi_{h}^{n+1}-\mathbf{q}_{h}\left(\phi_{h}^{n}\right)\right)\right) \cdot \nabla w d \mathbf{x} \\
& +\int_{\partial \Omega} S_{\epsilon}\left(\phi_{h}^{n+1}\right) w \mathbf{v}^{n+1} \cdot \mathbf{n} d \mathbf{s}=0 \quad \forall w \in X_{h} .
\end{aligned}
$$

We solve the non-linear system (31) using Newton's method. The Jacobian corresponding to $R\left(\phi_{h}, w\right)$ in (31) at the $k$-th Newton iteration is given by

$$
\begin{aligned}
\frac{\partial R\left(\phi_{h}^{k}, w_{i}\right)}{\partial \Phi_{j}}=: J_{i j}^{k} & =\int_{\Omega} S_{\epsilon}^{\prime}\left(\phi_{h}^{k}\right)\left[\frac{1}{\Delta t} w_{i} w_{j}-\nabla w_{i} \cdot\left(\mathbf{v}^{n+1} w_{j}\right)\right] d \mathbf{x}+\int_{\Omega} \lambda \nabla w_{i} \cdot \nabla w_{j} d \mathbf{x} \\
& +\int_{\partial \Omega} S_{\epsilon}^{\prime}\left(\phi_{h}^{k}\right) w_{i} w_{j} \mathbf{v}^{n+1} \cdot \mathbf{n} d \mathbf{s} .
\end{aligned}
$$

In Figure 3, we present numerical solutions of the vortex problem at $t=2$. The results shown in the middle panel were obtained using the proposed discretization (31). The implications of treating the advection term explicitly and penalization term implicitly are illustrated by the snapshots shown in the left and right panels, respectively. As expected based on the results of $\S 3.2$, the explicit treatment of the advection term gives rise to spurious distortions of the interface shape. The implicit treatment of the penalization term has hardly any visible influence on the accuracy of the results but significantly increases the computational effort associated with solving the non-linear system.

We close this section demonstrating the importance and effect of treating the penalization term via the $\mathcal{C}^{0}$ reconstruction proposed in $\S 4$, opposed to its non-projected counterpart in $\S 3$. To do this we solve the vortex problem at $t=2$ and 4 via model (15) and (24). When solving (15) we consider a single- and a double-well potential, see $\S 3.1$. In all cases we use a first-order approximation in time, treat the advection term implicitly and the penalization term explicitly. The results are shown in figure 4 . 

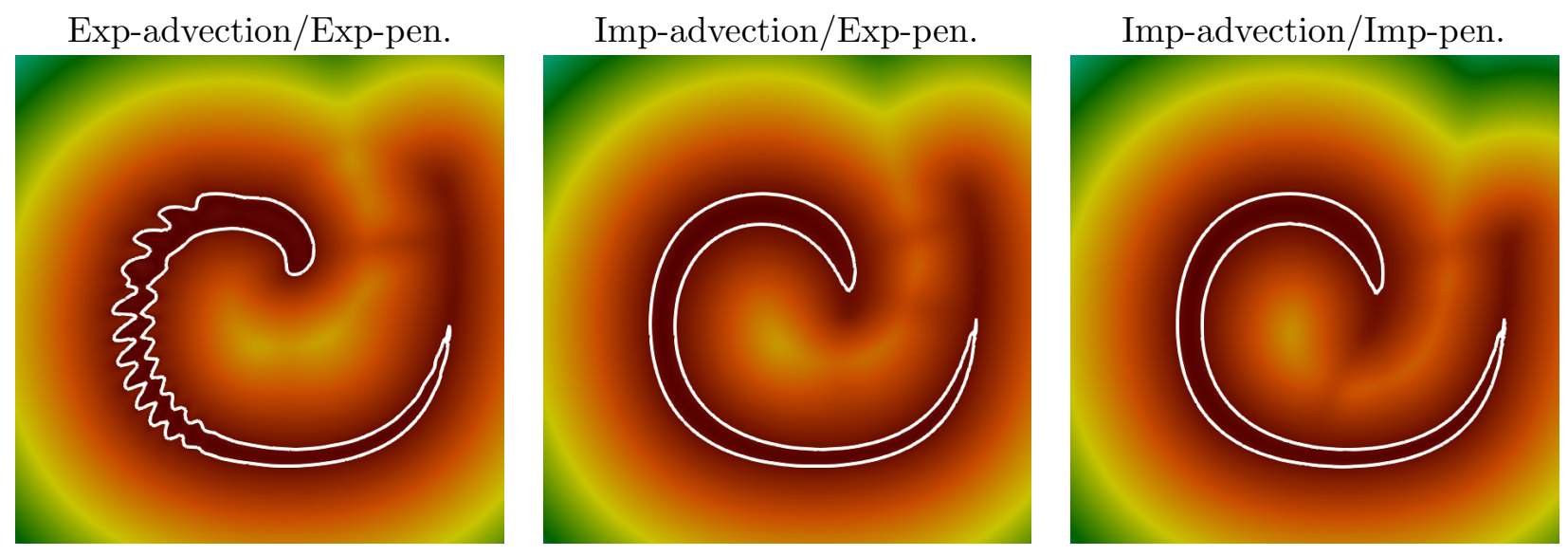

Figure 3: Solutions of the vortex problem at $t=2$ obtained using the monolithic conservative level set formulation (31) with $\lambda=0.1$. The advection term is treated explicitly in the left panel and implicitly elsewhere. The penalization term is treated implicitly in the right panel and explicitly elsewhere.

\subsection{Second-order time discretization}

To achieve second-order temporal accuracy, we discretize (24) in time using an implicit-explicit RungeKutta scheme proposed by Hundsdorfer and Verwer [18, §4.3]. The two-stage time integrator

$$
\begin{array}{r}
\frac{S_{\epsilon}\left(\phi^{*}\right)-S_{\epsilon}\left(\phi^{n}\right)}{\Delta t}+\nabla \cdot\left\{\frac{\mathbf{v}^{n} S_{\epsilon}\left(\phi^{n}\right)+\mathbf{v}^{n+1} S_{\epsilon}\left(\phi^{n+1}\right)}{2}-\lambda\left[\frac{\nabla \phi^{n}+\nabla \phi^{n+1}}{2}-\mathbf{q}\left(\phi^{n}\right)\right]\right\}=0, \\
\frac{S_{\epsilon}\left(\phi^{n+1}\right)-S_{\epsilon}\left(\phi^{n}\right)}{\Delta t}+\nabla \cdot\left\{\frac{\mathbf{v}^{n} S_{\epsilon}\left(\phi^{n}\right)+\mathbf{v}^{n+1} S_{\epsilon}\left(\phi^{n+1}\right)}{2}-\frac{\lambda}{2}\left(\nabla \phi^{n}+\nabla \phi^{n+1}-\left[\mathbf{q}\left(\phi^{n}\right)+\mathbf{q}\left(\phi^{*}\right)\right]\right)\right\}=0
\end{array}
$$

represents a predictor-corrector version of the second-order accurate Crank-Nicolson method. The predictor $\phi^{*} \approx \phi^{n+1}$ is used to approximate $\mathbf{q}\left(\phi^{n+1}\right) \approx \mathbf{q}\left(\phi^{*}\right)$ at the second stage. The discretization in space is performed using the continuous Galerkin method as in $\S 4.2$. In the context of high-order finite element approximations, we favor the use of Bernstein basis polynomials (see for instance Ainsworth et al. [1], Anderson et al. [2], Kirby [21], Lohmann et al. [27]) if the mass matrix is lumped during $L^{2}$ projections. In contrast to commonly employed Lagrange elements, the Bernstein basis functions are non-negative. This property guarantees that the weighted lumped mass matrix of the $\mathcal{C}^{0}$ normal reconstruction step (30) remains non-singular for polynomial spaces of arbitrary order. We remark that the use of mass lumping may degrade the accuracy of high-order finite element approximations, see $§ 5$. Numerical results for the two-dimensional vortex problem are presented in $\S 6.1$.

\section{Convergence properties}

In this section we explore the convergence properties of the method we propose. We start in $\S 5.1$ considering the elliptic part of (24). Then, in $\S 5.2$ we consider the full conservation law (24) using the discretization in $\S 4.3$.

\subsection{Convergence studies for the elliptic operator}

Given a velocity field, the transport equation, in general, does not preserve the distance function property. Therefore, it is unreasonable to expect full high-order convergence with respect to a distance function. Moreover, in applications to level-set methods we are mainly interested in the behavior near 
Model (15) via single-well pot.
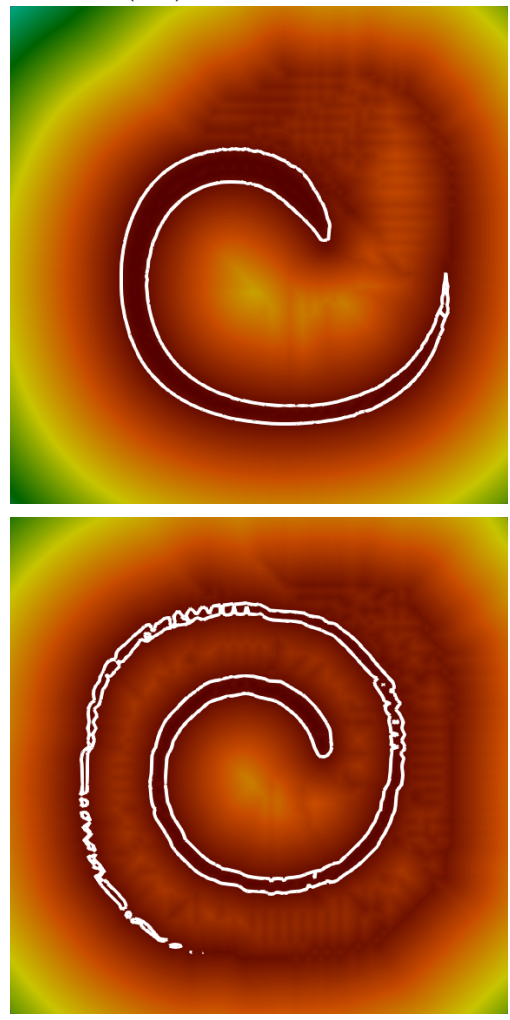

Model (15) via double-well pot.
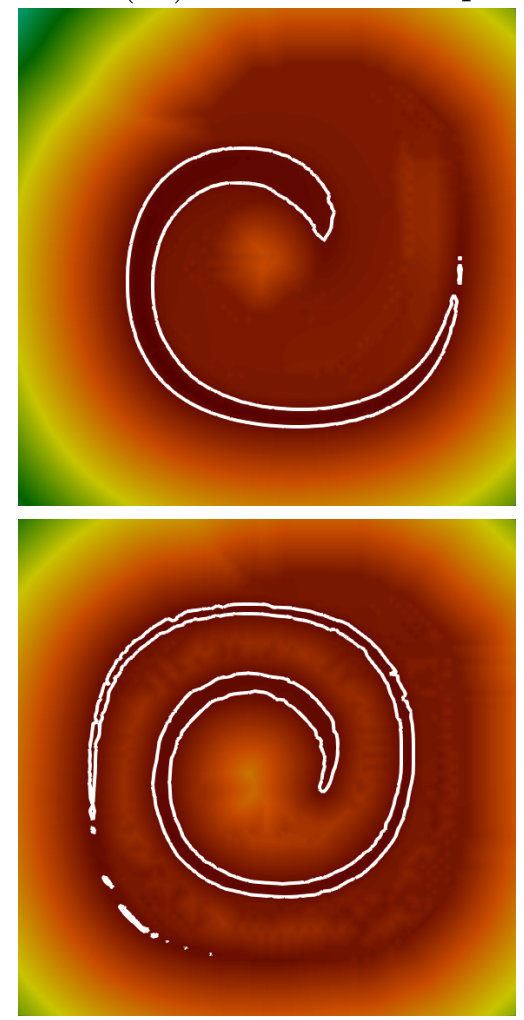

Model (24)
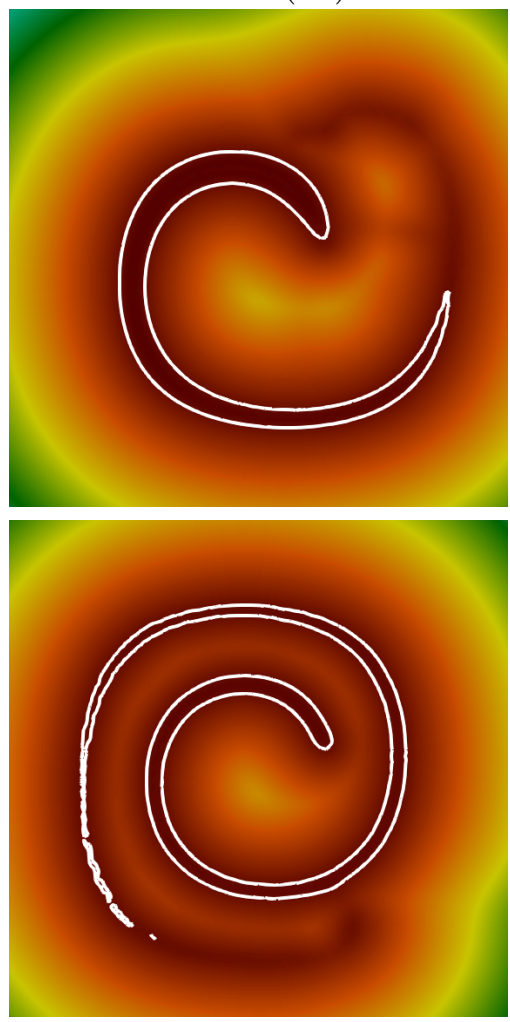

Figure 4: Solution of the vortex problem at (first row) $t=2$ and (second row) $t=4$. The first two columns correspond to model (15) using a single- and a double-well potential respectively. In the third column we consider model (24). In all experiments the advection term is treated implicitly and the penalization term explicitly.

the interface; i.e., near the zero-level set. Nevertheless, for certain velocity fields the exact solution is a distance function. In particular, in this section we consider $\mathbf{v}=0$ and perform a series of numerical experiments to study the convergence properties of the model we propose in this limited context.

We consider $\Omega=(0,1)^{2}$ and let $\phi(\mathbf{x})$ denote the (exact) signed distance function to a circle with radius $r=0.15$ centered at $\left(x_{c}, y_{c}\right)=(0.5,0.75)$, see (20a). In all experiments we use a first order approximation in time with $\Delta t=1$, treat the penalization term explicitly and compute the following metrics:

$$
\left\|\phi_{h}\left(\mathbf{x}, t^{1}\right)-\phi(\mathbf{x})\right\|_{L^{2}(\Omega)}, \quad \frac{1}{\left|\Omega^{*}\right|}\left\|\phi_{h}\left(\mathbf{x}, t^{1}\right)-\phi(\mathbf{x})\right\|_{L^{2}\left(\Omega^{*}\right)}, \quad\left\|H_{\epsilon}\left(\phi_{h}\left(\mathbf{x}, t^{1}\right)\right)-H_{\epsilon}(\phi(\mathbf{x}))\right\|_{L^{2}(\Omega)},
$$

where $\Omega^{*}:=\left\{\mathbf{x} \in \Omega|| \phi_{h}\left(\mathbf{x}, t^{1}\right) \mid \leq 2 \epsilon\right\}$ defines a neighborhood around the zero level-set.

We start with model (12); i.e., we solve

$$
\int_{\Omega}\left[S_{\epsilon}\left(\phi_{h}^{1}\right)-S_{\epsilon}\left(\phi_{h}^{0}\right)\right] w d \mathbf{x}+\int_{\Omega}\left[\lambda\left(\nabla \phi_{h}^{1}-\frac{\nabla \phi_{h}^{0}}{\left|\nabla \phi_{h}^{0}\right|}\right)\right] \cdot \nabla w d \mathbf{x}=0
$$

and obtain the results in table 1 considering linear and quadratic spaces $X_{h}^{1}$ and $X_{h}^{2}$ respectively, see $\S 2.1$. Note that the number of elements is adjusted to have the same number of degrees of freedom in each row of the table. Due to lack of regularity on $\phi(\mathbf{x})$, we loose the full high-order accuracy with respect to the $L^{2}(\Omega)$-norm. However, we recover it in a neighborhood of the zero-level set; i.e., we get full accuracy in the (normalized) $L^{2}\left(\Omega^{*}\right)$-norm. Also due to lack of regularity on the regularized Heaviside function we loose full accuracy on $\left\|H_{\epsilon}\left(\phi_{h}\left(\mathbf{x}, t^{1}\right)\right)-H_{\epsilon}(\phi(\mathbf{x}))\right\|_{L^{2}(\Omega)}$; however, it is clear the improvement by using high-order spaces. 


\begin{tabular}{|c|c|c|c|c|c|c|c|c|c|c|c|c|}
\hline & \multicolumn{4}{|c|}{$\left\|\phi_{h}\left(\mathbf{x}, t^{1}\right)-\phi(\mathbf{x})\right\|_{L^{2}(\Omega)}$} & \multicolumn{4}{|c|}{$\frac{1}{\mid \Omega^{*}}\left\|\phi_{h}\left(\mathbf{x}, t^{1}\right)-\phi(\mathbf{x})\right\|_{L^{2}\left(\Omega^{*}\right)}$} & \multicolumn{4}{|c|}{$\left\|H_{\epsilon}\left(\phi_{h}\left(\mathbf{x}, t^{1}\right)\right)-H_{\epsilon}(\phi(\mathbf{x}))\right\|_{L^{2}(\Omega)}$} \\
\hline N-DOFs & $X_{h}^{1}$-space & & $X_{h}^{2}$-space & & $X_{h}^{1}$-space & & $X_{h}^{2}$-space & & $X_{h}^{1}$-space & & $X_{h}^{2}$-space & \\
\hline 1681 & $1.71 \mathrm{E}-4$ & - & $9.27 \mathrm{E}-5$ & - & $1.91 \mathrm{E}-4$ & - & $6.68 \mathrm{E}-5$ & - & $5.52 \mathrm{E}-4$ & - & $1.58 \mathrm{E}-4$ & - \\
\hline 6561 & $4.85 \mathrm{E}-5$ & 1.82 & $1.04 \mathrm{E}-5$ & 3.15 & $3.99 \mathrm{E}-5$ & 2.26 & $6.36 \mathrm{E}-6$ & 3.39 & $1.90 \mathrm{E}-4$ & 1.53 & $2.76 \mathrm{E}-5$ & 2.51 \\
\hline 25921 & $1.33 \mathrm{E}-5$ & 1.86 & $2.60 \mathrm{E}-6$ & 1.99 & $9.31 \mathrm{E}-6$ & 2.09 & $6.90 \mathrm{E}-7$ & 3.20 & $6.67 \mathrm{E}-5$ & 1.51 & $4.75 \mathrm{E}-6$ & 2.53 \\
\hline 103041 & $3.60 \mathrm{E}-6$ & 1.88 & $6.49 \mathrm{E}-7$ & 1.99 & $2.30 \mathrm{E}-6$ & 2.01 & $8.35 \mathrm{E}-8$ & 3.04 & $2.35 \mathrm{E}-5$ & 1.50 & $8.54 \mathrm{E}-7$ & 2.47 \\
\hline 410881 & $9.60 \mathrm{E}-7$ & 1.90 & $1.62 \mathrm{E}-7$ & 1.99 & $5.72 \mathrm{E}-7$ & 2.00 & $1.04 \mathrm{E}-8$ & 3.01 & $8.31 \mathrm{E}-6$ & 1.50 & $1.50 \mathrm{E}-7$ & 2.50 \\
\hline
\end{tabular}

Table 1: Convergence on static problem with a non-projected penalization.

Now we consider model (24); i.e.,

$$
\int_{\Omega}\left[S_{\epsilon}\left(\phi_{h}^{1}\right)-S_{\epsilon}\left(\phi_{h}^{0}\right)\right] w d \mathbf{x}+\int_{\Omega}\left[\lambda\left(\nabla \phi_{h}^{1}-\mathbf{q}_{h}^{0}\right)\right] \cdot \nabla w d \mathbf{x}=0
$$

and compute $\mathbf{q}_{h}^{0}$ via a (weighted) lumped and consistent $L^{2}$ projection. The results are shown in tables 2 and 3 respectively. It is clear that using the lumped $L^{2}$ projection decreases the accuracy when high-order spaces are used.

\begin{tabular}{|c|c|c|c|c|c|c|c|c|c|c|c|c|}
\hline & \multicolumn{4}{|c|}{$\left\|\phi_{h}\left(\mathbf{x}, t^{1}\right)-\phi(\mathbf{x})\right\|_{L^{2}(\Omega)}$} & \multicolumn{4}{|c|}{$\frac{1}{\left|\Omega^{*}\right|}\left\|\phi_{h}\left(\mathbf{x}, t^{1}\right)-\phi(\mathbf{x})\right\|_{L^{2}\left(\Omega^{*}\right)}$} & \multicolumn{4}{|c|}{$\left\|H_{\epsilon}\left(\phi_{h}\left(\mathbf{x}, t^{1}\right)\right)-H_{\epsilon}(\phi(\mathbf{x}))\right\|_{L^{2}(\Omega)}$} \\
\hline N-DOFs & $X_{h}^{1}$-space & & $X_{h}^{2}$-space & & $X_{h}^{1}$-space & & $X_{h}^{2}$-space & & $X_{h}^{1}$-space & & $X_{h}^{2}$-space & \\
\hline 1681 & $8.58 \mathrm{E}-4$ & - & $1.01 \mathrm{E}-3$ & - & $7.14 \mathrm{E}-4$ & - & $6.90 \mathrm{E}-4$ & - & $6.23 \mathrm{E}-4$ & - & $4.51 \mathrm{E}-4$ & - \\
\hline 6561 & $2.39 \mathrm{E}-4$ & 1.84 & $2.75 \mathrm{E}-4$ & 1.88 & $7.07 \mathrm{E}-5$ & 3.33 & $6.85 \mathrm{E}-5$ & 3.33 & $1.91 \mathrm{E}-4$ & 1.70 & $1.00 \mathrm{E}-4$ & 2.17 \\
\hline 25921 & $6.50 \mathrm{E}-5$ & 1.87 & $7.44 \mathrm{E}-5$ & 1.88 & $1.14 \mathrm{E}-5$ & 2.63 & $8.67 \mathrm{E}-6$ & 2.98 & $6.42 \mathrm{E}-5$ & 1.57 & $2.56 \mathrm{E}-5$ & 1.96 \\
\hline 103041 & $1.74 \mathrm{E}-5$ & 1.90 & $1.98 \mathrm{E}-5$ & 1.90 & $2.36 \mathrm{E}-6$ & 2.27 & $1.27 \mathrm{E}-6$ & 2.76 & $2.24 \mathrm{E}-5$ & 1.51 & $8.22 \mathrm{E}-6$ & 1.64 \\
\hline 410881 & $4.61 \mathrm{E}-6$ & 1.91 & $5.24 \mathrm{E}-6$ & 1.92 & $5.55 \mathrm{E}-7$ & 2.08 & $2.35 \mathrm{E}-7$ & 2.43 & $7.90 \mathrm{E}-6$ & 1.50 & $2.80 \mathrm{E}-6$ & 1.55 \\
\hline
\end{tabular}

Table 2: Convergence on static problem with a lumped $L^{2}$-projected penalization.

\begin{tabular}{|c|c|c|c|c|c|c|c|c|c|c|c|c|}
\hline & \multicolumn{4}{|c|}{$\left\|\phi_{h}\left(\mathbf{x}, t^{1}\right)-\phi(\mathbf{x})\right\|_{L^{2}(\Omega)}$} & \multicolumn{4}{|c|}{$\frac{1}{\left|\Omega^{*}\right|}|| \phi_{h}\left(\mathbf{x}, t^{1}\right)-\phi(\mathbf{x}) \|_{L^{2}\left(\Omega^{*}\right)}$} & \multicolumn{4}{|c|}{$\left\|H_{\epsilon}\left(\phi_{h}\left(\mathbf{x}, t^{1}\right)\right)-H_{\epsilon}(\phi(\mathbf{x}))\right\|_{L^{2}(\Omega)}$} \\
\hline N-DOFs & $X_{h}^{1}$-space & & $X_{h}^{2}$-space & & $X_{h}^{1}$-space & & $X_{h}^{2}$-space & & $X_{h}^{1}$-space & & $X_{h}^{2}$-space & \\
\hline 1681 & $3.89 \mathrm{E}-4$ & - & $1.48 \mathrm{E}-4$ & - & $2.78 \mathrm{E}-4$ & - & $1.18 \mathrm{E}-4$ & - & $5.46 \mathrm{E}-4$ & - & $2.00 \mathrm{E}-4$ & - \\
\hline 6561 & $1.08 \mathrm{E}-4$ & 1.84 & $3.52 \mathrm{E}-5$ & 2.06 & $4.51 \mathrm{E}-5$ & 2.62 & $7.97 \mathrm{E}-6$ & 3.89 & $1.83 \mathrm{E}-4$ & 1.57 & $3.41 \mathrm{E}-5$ & 2.54 \\
\hline 25921 & $2.93 \mathrm{E}-5$ & 1.88 & $8.81 \mathrm{E}-6$ & 1.99 & $9.36 \mathrm{E}-6$ & 2.26 & $8.68 \mathrm{E}-7$ & 3.19 & $6.35 \mathrm{E}-5$ & 1.52 & $5.89 \mathrm{E}-6$ & 2.53 \\
\hline 103041 & $7.82 \mathrm{E}-6$ & 1.90 & $2.20 \mathrm{E}-6$ & 1.99 & $2.22 \mathrm{E}-6$ & 2.07 & $1.06 \mathrm{E}-7$ & 3.04 & $2.23 \mathrm{E}-5$ & 1.50 & $1.06 \mathrm{E}-6$ & 2.47 \\
\hline 410881 & $2.07 \mathrm{E}-6$ & 1.91 & $5.50 \mathrm{E}-7$ & 1.99 & $5.46 \mathrm{E}-7$ & 2.02 & $1.31 \mathrm{E}-8$ & 3.01 & $7.89 \mathrm{E}-6$ & 1.50 & $1.87 \mathrm{E}-7$ & 2.50 \\
\hline
\end{tabular}

Table 3: Convergence on static problem with a consistent $L^{2}$-projected penalization.

\subsection{Convergence studies for the conservation law}

We consider now the conservation law (24) with a full discretization given by (32) with continuous Galerkin finite elements and perform a convergence study, based on the metrics (33), using linear and quadratic spaces. This study is based on the vortex problem, see (20). The penalization term $\mathbf{q}_{h}$ is treated explicitly and is computed via a lumped and a consistent $L^{2}$ projection. In figure 5 we show the results at $t=4$ and in tables 4 and 5 we show the metrics for different refinement levels. The use of the consistent mass matrix in the weighted $L^{2}$ projection of $\nabla \phi_{h}$ leads to oscillations near the peak that propagate to the interface, distorting its shape and producing large numerical errors. This problem can be cured by lumping the mass matrix; however, as seen in $\S 5.1$, doing so degrades the convergence behavior of high-order finite element discretizations. The consistent-mass $L^{2}$ projection can be constrained to satisfy discrete maximum principles using the methodology presented by Kuzmin et al. [23]. The underlying limiting strategy was originally developed by Löhner [28] (pp. 257-260) building on the flux-corrected transport (FCT) algorithm of Boris and Book [10], Zalesak [42]. More 
advanced frame-invariant limiters specifically designed for continuous finite element approximations to vector fields can be found in Zeng and Scovazzi [43]. We do not explore these sophisticated approaches in the present work. In the context of level set methods, the simplest way to preserve high-order accuracy without violating discrete maximum principles is to use the consistent weighted element mass matrix in the narrow band $\Omega^{*}$ and its lumped counterpart elsewhere. In the rest of this work, we use linear finite elements and, therefore, compute the penalization term $\mathbf{q}_{h}$ using the fully lumped version of the weighted $L^{2}$ projection.

We close this section emphasizing that despite the problem discussed here, formulation (24) with the full discretization given by a continuous Galerkin finite element approximation to (32) recovers the expected accuracy of linear spaces and can be used with high-order spaces, provided the penalization $\mathbf{q}_{h}$ is computed via a lumped or constrained $L^{2}$ projection.

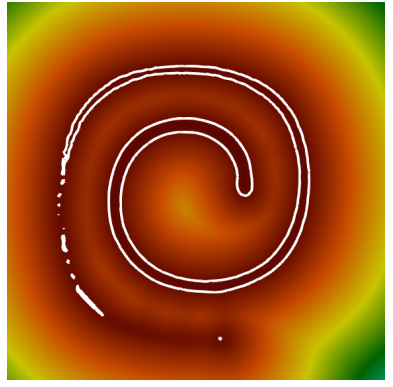

(a) Linear space $X_{h}^{1}$
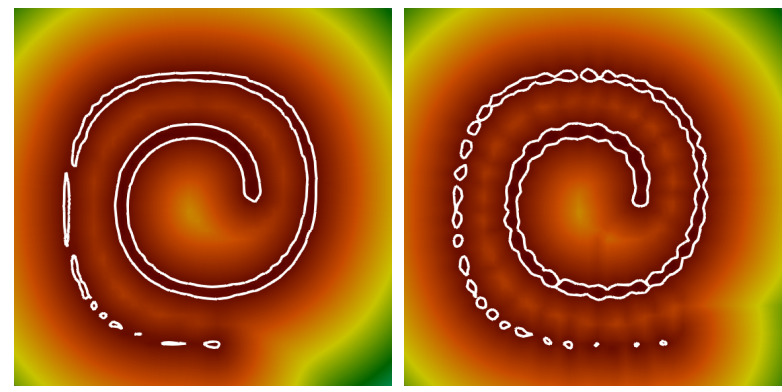

(b) Quadratic space $X_{h}^{2}$

Figure 5: Solution of the vortex problem at $t=4$ considering (a) linear and (b) quadratic spaces. For each space we consider the penalization term $\mathbf{q}_{h}$ via (left) a lumped and (right) a consistent $L^{2}$ projection. The number of cells is adjusted to have 6561 DOFs in all experiments.

\begin{tabular}{|c|c|c|c|c|c|c|c|c|c|c|c|c|}
\hline & \multicolumn{4}{|c|}{$\left\|\phi_{h}\left(\mathbf{x}, t^{1}\right)-\phi(\mathbf{x})\right\|_{L^{2}(\Omega)}$} & \multicolumn{4}{|c|}{$\frac{1}{\left|\Omega^{*}\right|}\left\|\phi_{h}\left(\mathbf{x}, t^{1}\right)-\phi(\mathbf{x})\right\|_{L^{2}\left(\Omega^{*}\right)}$} & \multicolumn{4}{|c|}{$\left\|H_{\epsilon}\left(\phi_{h}\left(\mathbf{x}, t^{1}\right)\right)-H_{\epsilon}(\phi(\mathbf{x}))\right\|_{L^{2}(\Omega)}$} \\
\hline N-DOFs & $X_{h}^{1}$-space & & $X_{h}^{2}$-space & & $X_{h}^{1}$-space & & $X_{h}^{2}$-space & & $X_{h}^{1}$-space & & $X_{h}^{2}$-space & \\
\hline 1681 & $9.37 \mathrm{E}-2$ & - & $1.12 \mathrm{E}-1$ & - & $1.03 \mathrm{E}-1$ & - & $1.22 \mathrm{E}-1$ & - & $2.31 \mathrm{E}-1$ & - & $2.48 \mathrm{E}-1$ & - \\
\hline 6561 & $1.98 \mathrm{E}-2$ & 2.24 & $2.25 \mathrm{E}-2$ & 2.31 & $2.16 \mathrm{E}-2$ & 2.25 & $2.42 \mathrm{E}-2$ & 2.33 & $9.92 \mathrm{E}-2$ & 1.21 & $1.07 \mathrm{E}-1$ & 1.21 \\
\hline 25921 & $5.05 \mathrm{E}-3$ & 1.96 & $5.36 \mathrm{E}-3$ & 2.06 & $5.10 \mathrm{E}-3$ & 2.08 & $5.46 \mathrm{E}-3$ & 2.14 & $3.63 \mathrm{E}-2$ & 1.44 & $3.87 \mathrm{E}-2$ & 1.46 \\
\hline
\end{tabular}

Table 4: Convergence with a lumped $L^{2}$-projected penalization.

\begin{tabular}{|c|c|c|c|c|c|c|c|c|c|c|c|c|}
\hline & \multicolumn{4}{|c|}{$\left\|\phi_{h}\left(\mathbf{x}, t^{1}\right)-\phi(\mathbf{x})\right\|_{L^{2}(\Omega)}$} & \multicolumn{4}{|c|}{$\frac{1}{\left|\Omega^{*}\right|}\left\|\phi_{h}\left(\mathbf{x}, t^{1}\right)-\phi(\mathbf{x})\right\|_{L^{2}\left(\Omega^{*}\right)}$} & \multicolumn{4}{|c|}{$\left\|H_{\epsilon}\left(\phi_{h}\left(\mathbf{x}, t^{1}\right)\right)-H_{\epsilon}(\phi(\mathbf{x}))\right\|_{L^{2}(\Omega)}$} \\
\hline N-DOFs & $X_{h}^{1}$-space & & $X_{h}^{2}$-space & & $X_{h}^{1}$-space & & $X_{h}^{2}$-space & & $X_{h}^{1}$-space & & $X_{h}^{2}$-space & \\
\hline 1681 & $1.06 \mathrm{E}-1$ & - & $9.98 \mathrm{E}-2$ & - & $8.49 \mathrm{E}-2$ & - & $7.09 \mathrm{E}-2$ & - & $1.85 \mathrm{E}-1$ & - & $1.50 \mathrm{E}-1$ & - \\
\hline 6561 & $6.91 \mathrm{E}-2$ & 0.61 & $1.23 \mathrm{E}-1$ & -0.30 & $4.06 \mathrm{E}-2$ & 1.06 & $7.58 \mathrm{E}-2$ & $\begin{array}{l}-0.09 \\
\end{array}$ & $8.08 \mathrm{E}-2$ & 1.19 & $6.06 \mathrm{E}-2$ & 1.30 \\
\hline 25921 & $9.81 \mathrm{E}-2$ & -0.50 & $1.01 \mathrm{E}-1$ & 0.28 & $6.24 \mathrm{E}-2$ & -0.62 & $6.28 \mathrm{E}-2$ & 0.27 & $3.39 \mathrm{E}-2$ & 1.25 & $2.13 \mathrm{E}-2$ & 1.50 \\
\hline
\end{tabular}

Table 5: Convergence with a consistent $L^{2}$-projected penalization.

\section{Numerical experiments}

In this section, we assess the numerical behavior of the two-step level set algorithm (32) in two and three space dimensions. We use linear finite elements and compute the penalization term $\mathbf{q}_{h}$ via a weighted lumped $L^{2}$ projection. Let $\phi$ be the exact solution to a given problem. For each experiment, 
we measure numerical errors using the following metrics:

$$
\begin{aligned}
\operatorname{LS}_{\mathrm{err}}\left(\phi_{h}\right) & =\frac{1}{\left|\Omega^{*}\right|}\left\|\phi(\mathbf{x})-\phi_{h}\left(\mathbf{x}, t^{n+1}\right)\right\|_{L^{2}\left(\Omega^{*}\right)}, \\
\operatorname{VOF}_{\mathrm{err}}\left(\phi_{h}\right) & =\frac{1}{L}\left\|H_{\epsilon}(\phi(\mathbf{x}))-H_{\epsilon}\left(\phi_{h}\left(\mathbf{x}, t^{n+1}\right)\right)\right\|_{L^{2}(\Omega)}, \\
\mathrm{I}_{\mathrm{err}}\left(\phi_{h}\right) & =\frac{1}{L}\left\|H_{\epsilon}(\phi(\mathbf{x}))-H_{\epsilon}\left(\phi_{h}\left(\mathbf{x}, t^{n+1}\right)\right)\right\|_{L^{1}(\Omega)}, \\
\mathrm{V}_{\mathrm{err}}\left(\phi_{h}\right) & =\frac{1}{\int_{\Omega} H\left(\phi_{h}(\mathbf{x}, 0)\right) d \mathbf{x}}\left|\int_{\Omega}\left[H\left(\phi_{h}(\mathbf{x}, 0)\right)-H\left(\phi_{h}(\mathbf{x}, t)\right)\right] d \mathbf{x}\right|, \\
\mathrm{V}_{\mathrm{err}}^{\epsilon}\left(\phi_{h}\right) & =\frac{1}{\int_{\Omega} H_{\epsilon}\left(\phi_{h}(\mathbf{x}, 0)\right) d \mathbf{x}}\left|\int_{\Omega}\left[H_{\epsilon}\left(\phi_{h}(\mathbf{x}, 0)\right)-H_{\epsilon}\left(\phi_{h}(\mathbf{x}, t)\right)\right] d \mathbf{x}\right|, \\
\mathrm{D}_{\mathrm{err}}\left(\phi_{h}\right) & =\frac{1}{2} \int_{\Omega}\left(\left|\nabla \phi_{h}\right|-1\right)^{2} d \mathbf{x}
\end{aligned}
$$

where $\Omega^{*}:=\left\{\mathrm{x} \in \Omega|| \phi_{h}\left(\mathrm{x}, t^{n+1}\right) \mid \leq 2 \epsilon\right\}, L$ is the $(d-1)$-dimensional measure of the zero level set $\Gamma(0)$; i.e., length in $2 \mathrm{D}$ and area in $3 \mathrm{D}, H_{\epsilon}(\cdot)$ is the smoothed Heaviside function defined in $\S 2.2$, and $H(\cdot)$ is the sharp Heaviside function

$$
H(z)= \begin{cases}0, & \text { if } z<0 \\ 0.5, & \text { if } z=0 \\ 1, & \text { if } z>0\end{cases}
$$

The quantity $\operatorname{LS}_{\text {err }}\left(\phi_{h}\right)$ measures the $L^{2}$ error in a neighborhood around the zero level set and $\operatorname{VOF}_{\text {err }}\left(\phi_{h}\right)$ measures the $L^{2}$ error of the volume fraction. The quantities $\mathrm{I}_{\text {err }}\left(\phi_{h}\right)$ and $\mathrm{V}_{\text {err }}\left(\phi_{h}\right)$ measure the extent of interface displacements and area/volume losses, see Enright et al. [14], Olsson and Kreiss [29]. The metric $\mathrm{D}_{\mathrm{err}}\left(\phi_{h}\right)$ measures the deviation of $\phi_{h}$ from a distance function. In all experiments of this section, we define $\lambda$ using (28) with $\tilde{\lambda}=1$.0. In addition, we set the tolerance of the non-linear solvers to $10^{-12}$ unless stated otherwise. When reporting the metrics we indicate the number of degrees of freedom as N-DOFs along with a characteristic mesh size denoted as $h$. All experiments in this section were performed and are available within the Proteus toolkit (http://proteustoolkit.org).

\subsection{Two-dimensional periodic vortex}

In the first two-dimensional test, we consider the periodic vortex problem (see Rider and Kothe [31]) again. The initial condition and the velocity field are given by (20). The domain of interest is the unit square $\Omega=(0,1)^{2}$. In Figure 6 , we show the numerical solutions and zero level sets obtained at $t=0,2,4,6$, and 8 using linear finite elements and 25,921 DOFs. The number of Newton iterations per stage (in (32)) is plotted in Figure 7 for three different tolerances in the stopping criteria of the non-linear solver. The error metrics defined by (34) are listed in Table 6. The initial length of the interface is $L \approx 9.42 \times 10^{-1}$ in this test.

\begin{tabular}{|c|c|c|c|c|c|c|c|}
\hline N-DOFs & $h$ & LS $_{\text {err }}$ & VOF $_{\text {err }}$ & $\mathrm{I}_{\text {err }}$ & $\mathrm{V}_{\text {err }}$ & $\mathrm{V}_{\text {err }}^{\epsilon}$ & $D_{\text {err }}$ \\
\hline 1,681 & $2.50 \mathrm{E}-2$ & $1.03 \mathrm{E}-1$ & $2.45 \mathrm{E}-1$ & $8.03 \mathrm{E}-2$ & $4.16 \mathrm{E}-2$ & $6.99 \mathrm{E}-12$ & $3.48 \mathrm{E}-3$ \\
\hline 6,561 & $1.25 \mathrm{E}-2$ & $2.16 \mathrm{E}-2$ & $1.05 \mathrm{E}-1$ & $1.97 \mathrm{E}-2$ & $8.48 \mathrm{E}-3$ & $6.41 \mathrm{E}-13$ & $4.00 \mathrm{E}-4$ \\
\hline 25,921 & $6.25 \mathrm{E}-3$ & $5.10 \mathrm{E}-3$ & $3.85 \mathrm{E}-2$ & $4.52 \mathrm{E}-3$ & $1.76 \mathrm{E}-3$ & $2.02 \mathrm{E}-12$ & $1.43 \mathrm{E}-4$ \\
\hline
\end{tabular}

Table 6: Error metrics (34) for the vortex problem solved using linear finite elements. The initial length of the interface is $L \approx 9.42 \times 10^{-1}$. 

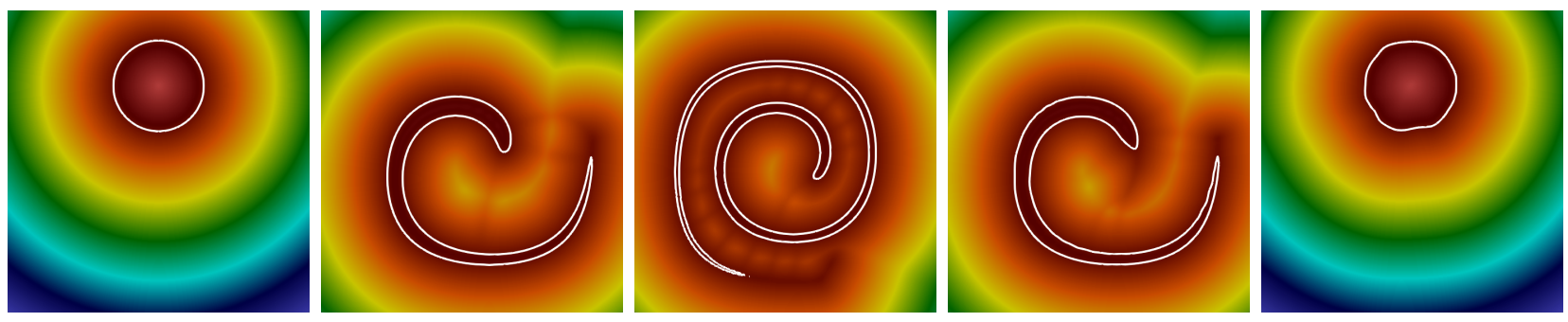

Figure 6: Approximate distance function $\phi_{h}$ and zero level set $\left\{\phi_{h}=0\right\}$ of the vortex problem at $t=0,2,4,6$, and 8. Solutions obtained using linear finite elements and 25,921 DOFs.
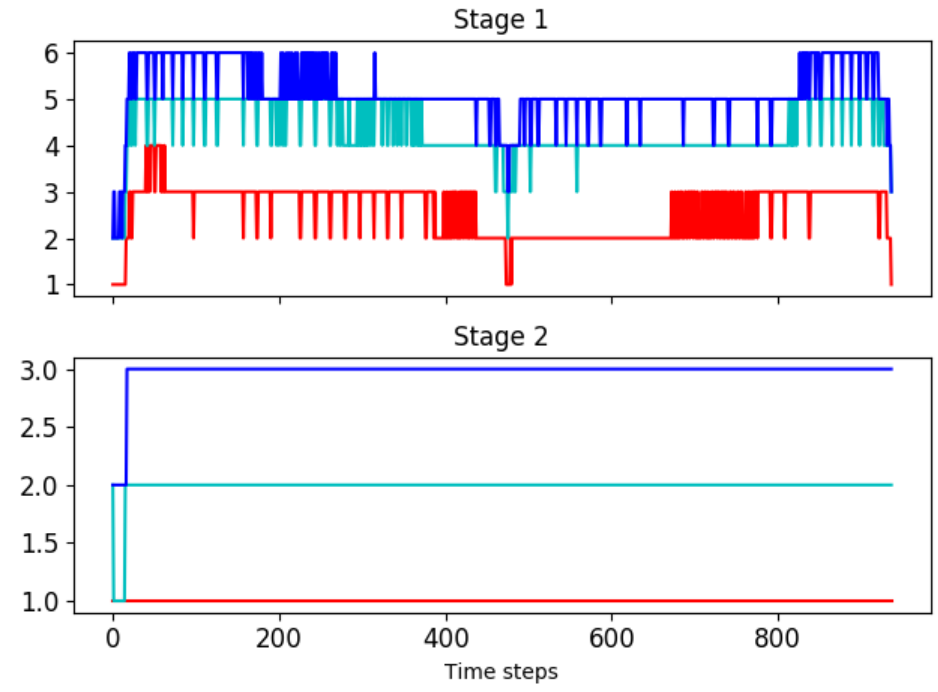

Figure 7: Number of Newton iterations for each stage in algorithm (32) discretized using linear finite elements with 6,561 DOFs. The iteration process is terminated when the tolerances (red) tol $=10^{-4}$, (cyan) tol $=10^{-8}$ and (blue) tol $=10^{-12}$ are satisfied.

\subsection{Solid body rotation of Zalesak's disk}

Another standard 2D benchmark is the slotted disc test proposed by Zalesak [42]. The computational domain is $\Omega=(0,1)^{2}$ again. The initial condition is given by the signed distance function

$$
\phi(\mathbf{x}, 0)= \pm \operatorname{dist}\left(\mathbf{x}, \Gamma_{0}\right)
$$

corresponding to the boundary $\Gamma_{0}=\partial Z$ of the set $Z=D \backslash\left\{(x, y) \in \Omega|| x-x_{c} \mid<0.025, y-y_{c}<0.1\right\}$, where $D$ is a disk of radius $r=0.15$ centered at $\left(x_{c}, y_{c}\right)=(0.5,0.75)$. We choose the positive distance in (35) if $\mathbf{x}=(x, y)$ is inside $\Gamma_{0}$ and the negative distance otherwise. The velocity field

$$
\mathbf{v}(x, y)=\left[\begin{array}{c}
-2 \pi(y-0.5) \\
2 \pi(x-0.5)
\end{array}\right]
$$

produces a solid body rotation around $(0.5,0.5)$. The exact solution of this problem preserves the shape of the interface and coincides with the initial condition after each full revolution.

In Figure 8, we show the solution and the zero level set at $t=0,0.2,0.4,0.6,0.8,1$ obtained using 25,921 DOFs. Figure 9 displays the zero level set at $t=1$ for three different refinement levels. The error metrics corresponding to the numerical results in Figure 9 are shown in Table 7 . The initial length of the interface is $L \approx 1.4380$ in this test. 

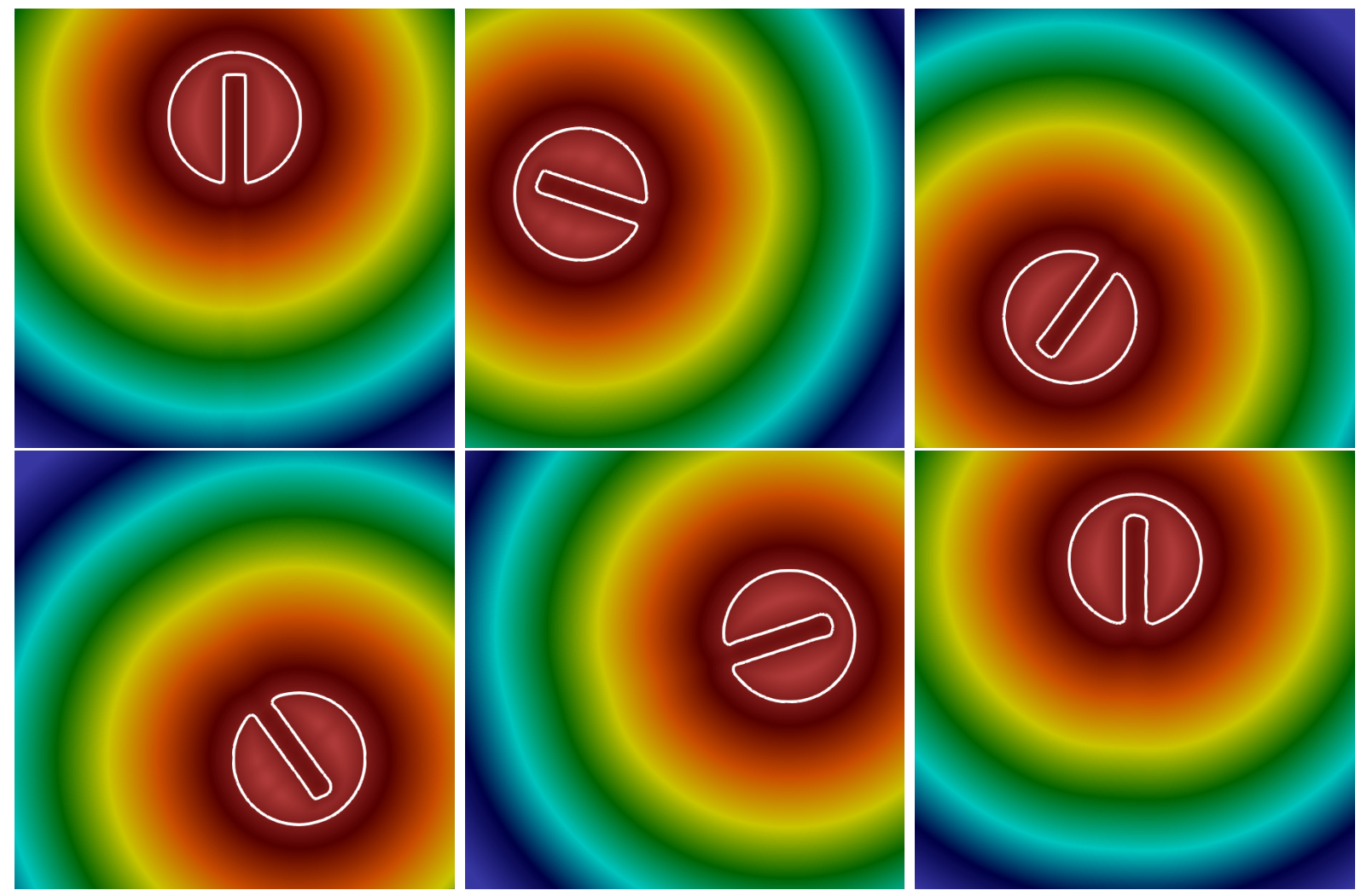

Figure 8: Approximate distance function $\phi_{h}$ and zero level set $\left\{\phi_{h}=0\right\}$ of Zalesak's disc at $t=$ $0,0.2,0.4,0.6,0.8$, and 1.0. Solutions obtained using linear finite elements with 25,921 DOFs.

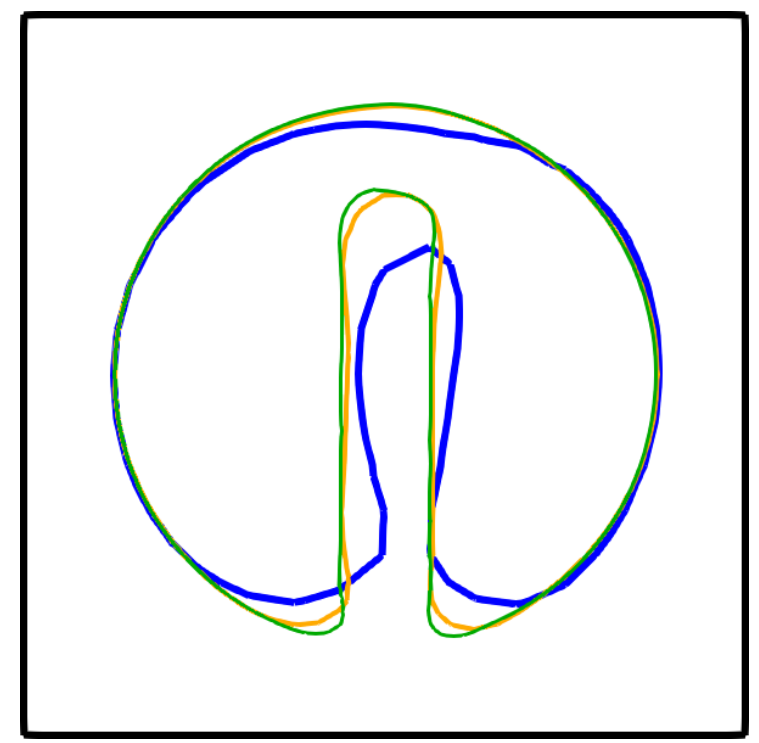

Figure 9: Zero level set $\left\{\phi_{h}=0\right\}$ of Zalesak's disk at $t=1$. Solutions obtained using linear finite elements. We consider three levels of refinement with (blue) 1,681, (orange) 6,561 and (green) 25,921 DOFs. 


\begin{tabular}{|c|c|c|c|c|c|c|c|}
\hline N-DOFs & $h$ & LS $_{\text {err }}$ & VOF $_{\text {err }}$ & $\mathrm{I}_{\text {err }}$ & $\mathrm{V}_{\text {err }}$ & $\mathrm{V}_{\text {err }}^{\epsilon}$ & $D_{\text {err }}$ \\
\hline 1,681 & $2.50 \mathrm{E}-2$ & $7.77 \mathrm{E}-3$ & $2.94 \mathrm{E}-2$ & $8.26 \mathrm{E}-3$ & $6.89 \mathrm{E}-2$ & $4.68 \mathrm{E}-16$ & $5.60 \mathrm{E}-3$ \\
\hline 6,561 & $1.25 \mathrm{E}-2$ & $2.88 \mathrm{E}-3$ & $1.20 \mathrm{E}-2$ & $1.42 \mathrm{E}-3$ & $4.39 \mathrm{E}-3$ & $1.33 \mathrm{E}-13$ & $2.75 \mathrm{E}-3$ \\
\hline 25,921 & $6.25 \mathrm{E}-3$ & $1.27 \mathrm{E}-3$ & $6.13 \mathrm{E}-3$ & $6.12 \mathrm{E}-4$ & $7.87 \mathrm{E}-4$ & $4.49 \mathrm{E}-13$ & $1.55 \mathrm{E}-3$ \\
\hline
\end{tabular}

Table 7: Error metrics (34) for Zalesak's disk problem solved using linear finite elements. The initial length of the interface is $L \approx 1.4380$.

\subsection{Three-dimensional solid body rotation}

Let us now consider a three-dimensional solid body rotation problem. The domain of interest is $\Omega=(0,1) \times(0,1) \times(0,0.5)$ and the initial condition is given by the signed distance function

$$
\phi(\mathbf{x}, 0)= \pm \operatorname{dist}(\mathbf{x}, 0),
$$

where $\Gamma_{0}=\left\{(x, y, z) \in \mathbb{R}^{3}:\left(x-x_{c}\right)^{2}+\left(y-y_{c}\right)^{2}+\left(z-z_{c}\right)^{2}=r^{2}\right\}$ is the surface of the sphere with radius $r=0.15$ centered at $\left(x_{c}, y_{c}, z_{c}\right)=(0.5,0.75,0.25)$. We choose the positive distance in (36) if $\mathbf{x}=(x, y, z)$ is inside the sphere and the negative distance otherwise. The velocity field

$$
\mathbf{v}(x, y, z)=\left[\begin{array}{c}
-2 \pi(y-0.5) \\
2 \pi(x-0.5) \\
0
\end{array}\right]
$$

corresponds to solid body rotation in the $x y$ plane. The exact solution coincides with the initial condition after each revolution. Computations are performed using linear finite elements with 67,626 DOFs. In Figure 10, we show the contour plots of the zero level sets at $t=0,0.2,0.4,0.6,0.8$, and 1 . The error metrics for three levels of mesh refinement are presented in Table 8.
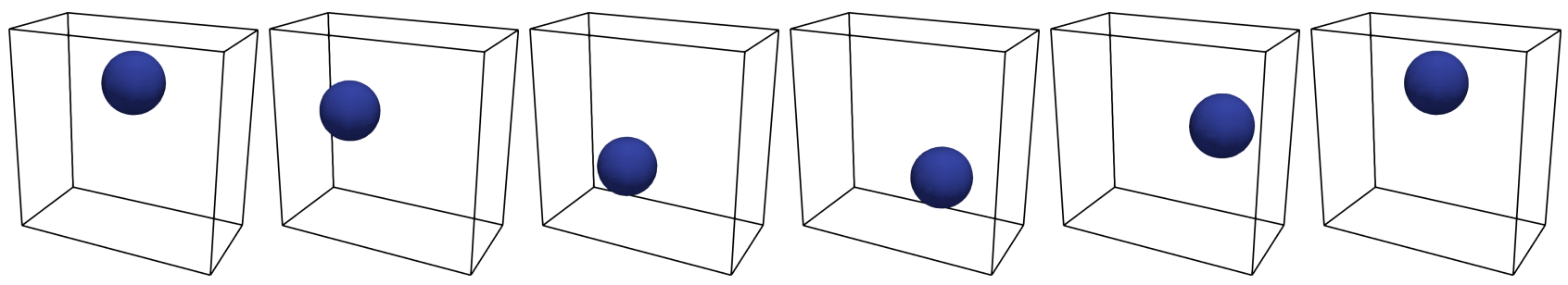

Figure 10: Zero level set $\left\{\phi_{h}=0\right\}$ representing the surface of the rotating sphere at $t=$ $0,0.2,0.4,0.6,0.8$, and 1. Solutions obtained using linear finite elements with 67,626 DOFs.

\begin{tabular}{|c|c|c|c|c|c|c|c|}
\hline N-DOFs & $h$ & LS $_{\text {err }}$ & VOF $_{\text {err }}$ & $\mathrm{I}_{\text {err }}$ & $\mathrm{V}_{\text {err }}$ & $\mathrm{V}_{\text {err }}^{\epsilon}$ & $D_{\text {err }}$ \\
\hline 1,183 & $8.33 \mathrm{E}-2$ & $1.70 \mathrm{E}-2$ & $7.02 \mathrm{E}-2$ & $7.38 \mathrm{E}-3$ & $9.68 \mathrm{E}-2$ & $6.95 \mathrm{E}-13$ & $1.95 \mathrm{E}-3$ \\
\hline 8,788 & $4.00 \mathrm{E}-2$ & $3.54 \mathrm{E}-3$ & $2.08 \mathrm{E}-2$ & $2.06 \mathrm{E}-3$ & $1.76 \mathrm{E}-3$ & $2.82 \mathrm{E}-14$ & $4.01 \mathrm{E}-4$ \\
\hline 67,626 & $2.00 \mathrm{E}-2$ & $8.25 \mathrm{E}-4$ & $7.43 \mathrm{E}-3$ & $5.19 \mathrm{E}-4$ & $1.13 \mathrm{E}-3$ & $2.32 \mathrm{E}-15$ & $1.75 \mathrm{E}-4$ \\
\hline
\end{tabular}

Table 8: Error metrics (34) for the 3D solid body rotation problem solved using linear finite elements on three different meshes. The initial interface area is $L \approx 2.82 \times 10^{-1}$. 


\subsection{Three-dimensional swirling flow}

In the last test, we consider the three-dimensional swirling flow problem proposed by LeVeque [25]. The computational domain is $\Omega=(0,1)^{3}$. The time-dependent velocity field is given by

$$
\mathbf{v}(x, y, z, t)=\left[\begin{array}{l}
2 \sin ^{2}(\pi x) \sin (2 \pi y) \sin (2 \pi z) \cos (\pi t / T) \\
-\sin (2 \pi x) \sin ^{2}(\pi y) \sin (2 \pi z) \cos (\pi t / T) \\
-\sin (2 \pi x) \sin (2 \pi y) \sin ^{2}(\pi z) \cos (\pi t / T)
\end{array}\right],
$$

where $T=3$ is the time corresponding to one cycle of swirling deformation. The initial condition

$$
\phi(\mathbf{x}, 0)= \pm \operatorname{dist}\left(\mathbf{x}, \Gamma_{0}\right)
$$

is the signed distance function of $\Gamma_{0}=\left\{(x, y, z) \in \mathbb{R}^{3}:\left(x-x_{c}\right)^{2}+\left(y-y_{c}\right)^{2}+\left(z-z_{c}\right)^{2}=r^{2}\right\}$, where $r=0.15$ as in our first 3D test. The center of the sphere enclosed by the interface $\Gamma_{0}$ is now placed at $\left(x_{c}, y_{c}, z_{c}\right)=(0.5,0.75,0.25)$. We choose the positive distance in (37) if $\mathbf{x}=(x, y, z)$ is inside the so-defined sphere and the negative distance otherwise. This problem is similar in nature to the $2 \mathrm{D}$ vortex problem considered in $\S 6.1$. The initial condition is deformed under the action of the periodically changing velocity field. At the time $t=T / 2$, the zero level set undergoes its maximum deformation producing thin filaments that are difficult to resolve unless local mesh refinement is performed in the interface region. As simulation continues, the interface flows back to its original position and the exact solution coincides with the initial data at the final time $t=T$. In Figure 11, we present numerical solutions obtained using linear finite elements on a uniform mesh with spacing $h=\Delta x=\Delta y=\Delta z=10^{-2}$, and in table 9 we list the metrics (34) for three levels of refinement. For this particular problem we use $\epsilon=\frac{1}{2} h(\mathbf{x})$, see $\S 2.2$. The reason is to improve the representation of the Heaviside and sign functions near the zones of maximum deformation where the thin filaments are likely to be under resolved.
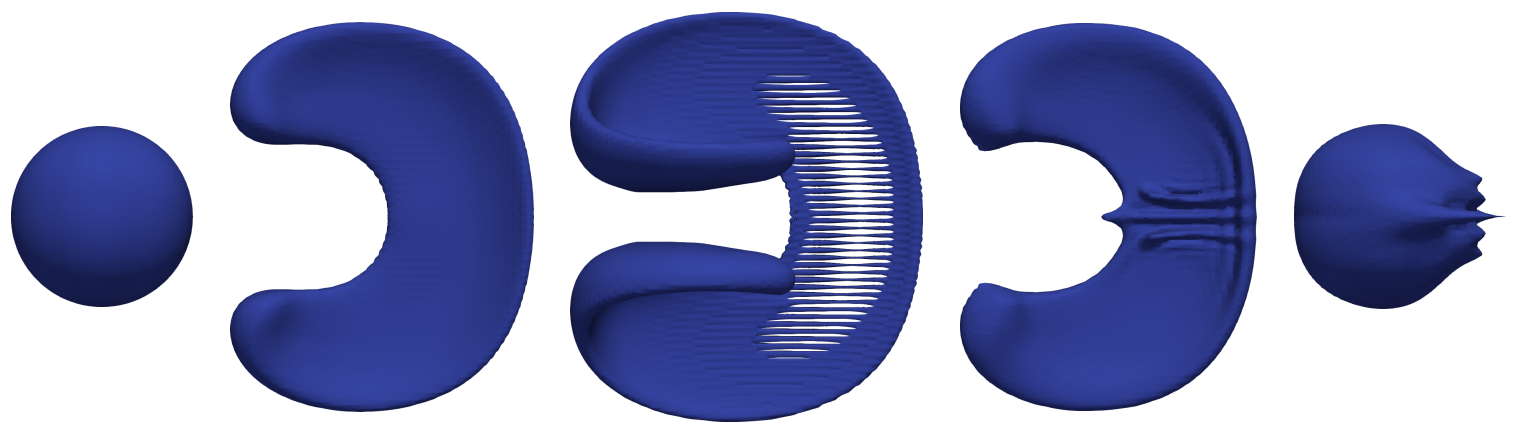

Figure 11: Zero level set $\left\{\phi_{h}=0\right\}$ representing the surface of the distorted sphere at $t=$ $0,0.7,1.5,2.3$, and 3. Solutions obtained using linear finite elements with 1,030,301 DOFs.

\begin{tabular}{|c|c|c|c|c|c|c|c|}
\hline N-DOFs & $h$ & LS $_{\text {err }}$ & VOF $_{\text {err }}$ & $\mathrm{I}_{\text {err }}$ & $\mathrm{V}_{\text {err }}$ & $\mathrm{V}_{\text {err }}^{\epsilon}$ & $D_{\text {err }}$ \\
\hline 17,576 & $4.00 \mathrm{E}-2$ & $1.48 \mathrm{E}-1$ & $3.55 \mathrm{E}-1$ & $5.08 \mathrm{E}-2$ & $5.86 \mathrm{E}-2$ & $3.27 \mathrm{E}-12$ & $4.26 \mathrm{E}-3$ \\
\hline 132,651 & $2.00 \mathrm{E}-2$ & $9.53 \mathrm{E}-2$ & $2.27 \mathrm{E}-1$ & $2.27 \mathrm{E}-2$ & $1.09 \mathrm{E}-2$ & $2.57 \mathrm{E}-11$ & $1.69 \mathrm{E}-3$ \\
\hline $1,030,301$ & $1.00 \mathrm{E}-2$ & $2.61 \mathrm{E}-2$ & $1.16 \mathrm{E}-1$ & $6.87 \mathrm{E}-3$ & $1.05 \mathrm{E}-3$ & $5.34 \mathrm{E}-11$ & $1.26 \mathrm{E}-3$ \\
\hline
\end{tabular}

Table 9: Error metrics (34) for the 3D swirling flow solved using linear finite elements on three different meshes. The initial interface area is $L \approx 2.82 \times 10^{-1}$. 


\section{Conclusions}

The main result of this work is the monolithic level set method (24) with the normal reconstruction $\mathbf{q}_{h}$ computed via the lumped $L^{2}$ projection (29) and (30). The proposed level set algorithm incorporates an elliptic redistancing term into a local conservation law leading to a non-linear transport equation for an approximate distance function. In contrast to most existing level set methods and their combinations with volume of fluid approaches, no additional post-processing or stabilization of advective terms is required. Moreover, the use of $C^{0}$ normal reconstructions eliminates convergence problems caused by the presence of local extrema or flat regions. The presented numerical studies indicate that the approximation of level set functions using high-order finite elements does not offer any significant benefits compared to the use of linear elements. The main reason is loss of accuracy due to mass lumping during the $C^{0}$ normal reconstruction. This problem can be cured via the use of a consistent $L^{2}$ projection and flux limiting strategies. In addition, the use of regularized Heaviside functions and/or piecewise-linear interface reconstructions imposes an order barrier on the overall accuracy w.r.t. interface shape and mass conservation properties. This drawback can be cured using different kinds of local mesh adaptation or enrichment of finite element spaces in elements containing the interface. In particular, level-set-aligned meshes can be generated using the mesh optimization procedures developed by Basting and Weismann [8].

Potential research directions to improve the results presented in this work include the use of limiting strategies to obtain non-oscillatory, high-order $\mathcal{C}^{0}$ reconstructions of the penalization term for redistancing; use of composite quadratures, see for instance Tornberg [37, §4.2], or more advance integration techniques for surface integrals, see for instance Engquist et al. [13], to improve the numerical integration of characteristic-like functions; use of optimal control methods, as in Basting and Kuzmin [7], to automatically select the parameter $\lambda$ and use of higher-order time integration techniques.

\section{Acknowledgments}

Manuel Quezada de Luna was supported by the US Army Coastal and Hydraulics Laboratory Research Participation. Permission was granted by the Chief of Engineers, US Army Corps of Engineers, to publish this information.

\section{References}

[1] M. Ainsworth, G. Andriamaro, and O. Davydov. Bernstein-Bèzier finite elements of arbitrary order and optimal assembly procedures. SIAM Journal on Scientific Computing, 33(6):3087-3109, 2011.

[2] R. Anderson, V. Dobrev, T. Kolev, D. Kuzmin, and M. Quezada de Luna. High-order local maximum principle preserving (MPP) discontinuous Galerkin finite element method for the transport equation. Journal of Computational Physics, 334(1):102-124, 2017.

[3] R. F. Ausas, E. A. Dari, and G. C. Buscaglia. A geometric mass-preserving redistancing scheme for the level set function. International Journal for Numerical Methods in Fluids, 65(8):989-1010, 2011.

[4] S. Badia and J. Bonilla. Monotonicity-preserving finite element schemes based on differentiable nonlinear stabilization. Computer Methods in Applied Mechanics and Engineering, 313:133-158, 2017.

[5] G. R. Barrenechea, V. John, and P. Knobloch. Analysis of algebraic flux correction schemes. SIAM Journal on Numerical Analysis, 54(4):2427-2451, 2016. 
[6] C. Basting and D. Kuzmin. A minimization-based finite element formulation for interfacepreserving level set reinitialization. Computing, 95(1):13-25, 2013.

[7] C. Basting and D. Kuzmin. Optimal control for mass conservative level set methods. Journal of Computational and Applied Mathematics, 270:343-352, 2014.

[8] S. Basting and M. Weismann. A hybrid level set/front tracking approach for finite element simulations of two-phase flows. Journal of Computational and Applied Mathematics, 270:471483, 2014.

[9] A. Bonito, J.-L. Guermond, and S. Lee. Numerical simulations of bouncing jets. International Journal for Numerical Methods in Fluids, 80(1):53-75, 2016.

[10] J. P. Boris and D. L. Book. Flux-corrected transport. I. SHASTA, a fluid transport algorithm that works. Journal of Computational Physics, 11(1):38-69, 1973.

[11] A. N. Brooks and T. J. Hughes. Streamline upwind/Petrov-Galerkin formulations for convection dominated flows with particular emphasis on the incompressible Navier-Stokes equations. Computer Methods in Applied Mechanics and Engineering, 32(1-3):199-259, 1982.

[12] T. F. Chan, G. H. Golub, and P. Mulet. A nonlinear primal-dual method for total variation-based image restoration. SIAM Journal on Scientific Computing, 20(6):1964-1977, 1999.

[13] B. Engquist, A.-K. Tornberg, and R. Tsai. Discretization of dirac delta functions in level set methods. Journal of Computational Physics, 207(1):28-51, 2005.

[14] D. Enright, R. Fedkiw, J. Ferziger, and I. Mitchell. A hybrid particle level set method for improved interface capturing. Journal of Computational Physics, 183(1):83-116, 2002.

[15] A. Ern and J.-L. Guermond. Theory and Practice of Finite Elements, volume 159. Springer Science \& Business Media, 2013.

[16] R. Fedkiw and S. Osher. Level Set Methods and Dynamic Implicit Surfaces, volume 44. Springer, 2002.

[17] C. W. Hirt and B. D. Nichols. Volume of fluid (VOF) method for the dynamics of free boundaries. Journal of Computational Physics, 39(1):201-225, 1981.

[18] W. Hundsdorfer and J. G. Verwer. Numerical Solution of Time-Dependent Advection-Diffusion-Reaction Equations, volume 33. Springer Science \& Business Media, 2013.

[19] S. Ianniello and A. Di Mascio. A self-adaptive oriented particles level-set method for tracking interfaces. Journal of Computational Physics, 229(4):1353-1380, 2010.

[20] C. E. Kees, I. Akkerman, M. W. Farthing, and Y. Bazilevs. A conservative level set method suitable for variable-order approximations and unstructured meshes. Journal of Computational Physics, 230(12):4536-4558, 2011.

[21] R. C. Kirby. Fast simplicial finite element algorithms using Bernstein polynomials. Numerische Mathematik, 117(4):3087-3109, 2011.

[22] D. Kuzmin. An optimization-based approach to enforcing mass conservation in level set methods. Journal of Computational and Applied Mathematics, 258:78-86, 2014. 
[23] D. Kuzmin, M. Möller, J. N. Shadid, and M. Shashkov. Failsafe flux limiting and constrained data projections for equations of gas dynamics. Journal of Computational Physics, 229(23):8766-8779, 2010.

[24] A.-C. Lesage and A. Dervieux. Conservation correction by dual level set. Research report, INRIA, 2009. URL http://hal.inria.fr/inria-00430191/en/.

[25] R. J. LeVeque. High-resolution conservative algorithms for advection in incompressible flow. SIAM Journal on Numerical Analysis, 33(2):627-665, 1996.

[26] C. Li, C. Xu, C. Gui, and M. D. Fox. Distance regularized level set evolution and its application to image segmentation. IEEE Transactions on Image Processing, 19(12):3243-3254, 2010.

[27] C. Lohmann, D. Kuzmin, J. N. Shadid, and S. Mabuza. Flux-corrected transport algorithms for continuous Galerkin methods based on high order Bernstein finite elements. Journal of Computational Physics, 344(1):51-186, 2017.

[28] R. Löhner. Applied CFD Techniques: An Introduction Based on Finite Element Methods. John Wiley \& Sons, 2008.

[29] E. Olsson and G. Kreiss. A conservative level set method for two phase flow. Journal of Computational Physics, 210(1):225-246, 2005.

[30] S. Osher and J. A. Sethian. Fronts propagating with curvature-dependent speed: algorithms based on hamilton-jacobi formulations. Journal of Computational Physics, 79(1):12-49, 1988.

[31] W. J. Rider and D. B. Kothe. Stretching and tearing interface tracking methods. AIAA paper, 95:1-11, 1995.

[32] J. A. Sethian. A fast marching level set method for monotonically advancing fronts. Proceedings of the National Academy of Sciences, 93(4):1591-1595, 1996.

[33] J. A. Sethian. Level Set Methods and Fast Marching Methods: Evolving Interfaces in Computational Geometry, Fluid Mechanics, Computer Vision, and Materials Science, volume 3. Cambridge University Press, 1999.

[34] A. Smolianski. Numerical Modeling of Two-Fluid Interfacial Flows. PhD thesis, University of Jyväskylä, 1999.

[35] M. Sussman and E. G. Puckett. A coupled level set and volume-of-fluid method for computing 3D and axisymmetric incompressible two-phase flows. Journal of Computational Physics, 162(2): 301-337, 2000.

[36] M. Sussman, P. Smereka, and S. Osher. A level set approach for computing solutions to incompressible two-phase flow. Journal of Computational Physics, 114(1):146-159, 1994.

[37] A.-K. Tornberg. Multi-dimensional quadrature of singular and discontinuous functions. BIT Numerical Mathematics, 42(3):644-669, 2002.

[38] M. K. Touré and A. Soulaïmani. Stabilized finite element methods for solving the level set equation without reinitialization. Computers \& Mathematics with Applications, 71(8):1602-1623, 2016.

[39] Y.-H. R. Tsai, L.-T. Cheng, S. Osher, and H.-K. Zhao. Fast sweeping algorithms for a class of hamilton-jacobi equations. SIAM Journal on Numerical Analysis, 41(2):673-694, 2003.

[40] T. Utz, F. Kummer, and M. Oberlack. Interface-preserving level-set reinitialization for dg-fem. International Journal for Numerical Methods in Fluids, 84(4):183-198, 2017. 
[41] L. Ville, L. Silva, and T. Coupez. Convected level set method for the numerical simulation of fluid buckling. International Journal for Numerical Methods in Fluids, 66(3):324-344, 2011.

[42] S. T. Zalesak. Fully multidimensional flux-corrected transport algorithms for fluids. Journal of Computational Physics, 31(3):335-362, 1979.

[43] X. Zeng and G. Scovazzi. A frame-invariant vector limiter for flux corrected nodal remap in arbitrary lagrangian-eulerian flow computations. Journal of Computational Physics, 270(1):753$783,2014$. 